This item was submitted to Loughborough's Research Repository by the author.

Items in Figshare are protected by copyright, with all rights reserved, unless otherwise indicated.

\title{
Content placement in cache-enabled sub-6 GHz and millimeter-wave multi- antenna dense small cell networks
}

PLEASE CITE THE PUBLISHED VERSION

https://doi.org/10.1109/TWC.2018.2794368

PUBLISHER

IEEE

VERSION

VoR (Version of Record)

\section{PUBLISHER STATEMENT}

This work is made available according to the conditions of the Creative Commons Attribution 3.0 Unported (CC BY 3.0) licence. Full details of this licence are available at: http://creativecommons.org/licenses/by/3.0/

\section{LICENCE}

CC BY 3.0

\section{REPOSITORY RECORD}

Zhu, Yongxu, Gan Zheng, Lifeng Wang, Kai-Kit Wong, and Liqiang Zhao. 2019. "Content Placement in Cacheenabled Sub-6 Ghz and Millimeter-wave Multi-antenna Dense Small Cell Networks”. figshare.

https://hdl.handle.net/2134/33787. 


\title{
Content Placement in Cache-Enabled Sub-6 GHz and Millimeter-Wave Multi-Antenna Dense Small Cell Networks
}

\author{
Yongxu Zhu ${ }^{\circledR}$, Gan Zheng ${ }^{\circledR}$, Senior Member, IEEE, Lifeng Wang ${ }^{\circledR}$, Member, IEEE, \\ Kai-Kit Wong, Fellow, IEEE, and Liqiang Zhao ${ }^{\circledR}$, Member, IEEE
}

\begin{abstract}
This paper studies the performance of cacheenabled dense small cell networks consisting of multi-antenna sub-6 GHz and millimeter-wave (mm-wave) base stations. Different from the existing works which only consider a single antenna at each base station, the optimal content placement is unknown when the base stations have multiple antennas. We first derive the successful content delivery probability by accounting for the key channel features at sub-6 $\mathrm{GHz}$ and mm-wave frequencies. The maximization of the successful content delivery probability is a challenging problem. To tackle it, we first propose a constrained cross-entropy algorithm which achieves the near-optimal solution with moderate complexity. We then develop another simple yet effective heuristic probabilistic content placement scheme, termed two-stair algorithm, which strikes a balance between caching the most popular contents and achieving content diversity. Numerical results demonstrate the superior performance of the constrained cross-entropy method and that the two-stair algorithm yields significantly better performance than only caching the most popular contents. The comparisons between the sub-6 GHz and mm-wave systems reveal an interesting tradeoff between caching capacity and density for the mm-wave system to achieve similar performance as the sub-6 GHz system.
\end{abstract}

Index Terms-Sub-6 GHz, millimeter wave, caching placement, user association, dense networks.

\section{INTRODUCTION}

$\mathbf{T}$ HE global mobile data traffic continues growing at an unprecedented pace and will reach 49 exabytes monthly by 2021, of which 78 percent will be video contents [2]. To meet the high capacity requirement for the future mobile networks, one promising solution is network densification, i.e., deploying dense small cell base stations (SBSs) in the

Manuscript received July 9, 2017; revised November 5, 2017; accepted January 9, 2018. Date of publication February 9, 2018; date of current version May 8, 2018. This work was supported by the U.K. Engineering and Physical Sciences Research Council under Grant EP/N007840/1 and Grant EP/N008219/1 and in part by the National Natural Science Foundation of China under Grant 61771358. This paper was presented at the IEEE Global Telecommunications Conference (GLOBECOM), Singapore, December 2017 [1]. The associate editor coordinating the review of this paper and approving it for publication was S. Mukherjee. (Corresponding author: Yongxu Zhu)

Y. Zhu and G. Zheng are with the Wolfson School of Mechanical, Electrical and Manufacturing Engineering, Loughborough University, Loughborough LE11 3TU, U.K. (e-mail: y.zhu4@lboro.ac.uk; g.zheng@lboro.ac.uk).

L. Wang and K.-K. Wong are with the Department of Electronic and Electrical Engineering, University College London, London WC1E 6BT, U.K. (e-mail: lifeng.wang@ucl.ac.uk; kai-kit.wong@ucl.ac.uk).

L. Zhao is with the State Key Laboratory of Integrated Service Networks, Xidian University, Xi'an 710071, China (e-mail: lqzhao@mail.xidian.edu.cn).

Color versions of one or more of the figures in this paper are available online at http://ieeexplore.ieee.org.

Digital Object Identifier 10.1109/TWC.2018.2794368 existing macrocell cellular networks. Although large numbers of small cells shorten the communication distance, the major challenge is to transfer the huge amount of mobile data from the core networks to the small cells and this imposes stringent demands on backhaul links. To address this problem, caching popular contents at small cells has been proposed as one of the most effective solutions, considering the fact that most mobile data are contents such as video, weather forecasts, news and maps, that are repeatedly requested and cacheable [3]. The combination of small cells and caching will bring content closer to users, decrease backhaul traffic and reduce transmission delays, thus alleviating many bottleneck problems in wireless content delivery networks. This paper focuses on the caching design at both sub- $6 \mathrm{GHz}$ ( $\mu$-wave) and $\mathrm{mm}$-wave (mm-wave) ${ }^{1}$ SBSs in dense small cell networks.

\section{A. Related Works}

1) Caching in $\mu$-wave and mm-wave Networks: mm-wave communication has received much interest for providing high capacity because there are vast amount of inexpensive spectra available in the $30 \mathrm{GHz}-300 \mathrm{GHz}$ range. However, compared to $\mu$-wave frequencies, mm-wave channel experiences excessive attenuation due to rainfall, atmospheric or gaseous absorption, and is susceptible to blockage. To redeem these drawbacks, mm-wave small cells need to adopt narrow beamforming and be densely deployed in an attempt to provide seemless coverage [4]-[6]. The study of content caching applications in mm-wave networks is of great importance, due to the fact that mm-wave will be a key component of future wireless access and content caching at the edge of networks is one of $5 \mathrm{G}$ service requirements [6]. Cache assignment with video streaming in mm-wave SBSs on the highway is discussed in [7] and it is shown to significantly reduce the connection and retrieval delays. Certainly, combining the advantages of $\mu$-wave and mm-wave technologies will bring more benefits [8]. Caching in dual-mode SBSs that integrate both $\mu$-wave and mm-wave frequencies is studied in [9], where dynamic matching game-theoretic approach is applied to maximize the handovers to SBSs in the mobility management scenarios. The proposed methods can minimize handover failures and reduce energy consumption in highly mobile heterogeneous networks. Dynamic traffic in cacheenabled network was studied in [10].

\footnotetext{
${ }^{1}$ In this paper, we focus on mm-wave frequencies from $30 \mathrm{GHz}$ to $300 \mathrm{GHz}$.
} 
Recent contributions also pay attention to the caching in MIMO networks such as [11]-[13]. In [11] and [12], cacheenabled cooperative MIMO framework for wireless video streaming is investigated. In [13], coded caching for downlink MIMO channel is discussed.

2) Optimization of Content Placement: Content placement with finite cache size is the key issue in caching design, since unplanned caching in nearby SBSs will result in more interference. The traditional method of caching most popular content (MPC) in wired networks is no longer optimal when considering the wireless transmission. A strategy that combines MPC and the largest content diversity caching is proposed in [14], together with cooperative transmission in cluster-centric small cell networks. This strategy is extended to the distributed relay networks with relay clustering in [15] to combat the halfduplex constraint, and it significantly improves the outage performance. A multi-threshold caching that allows BSs to store different number of copies of contents according to their popularity is proposed in [16], and it allows a finer partitioning of the cache space than binary threshold, but its complexity is exponential in the number of thresholds.

Probabilistic content placement under random network topologies has also been investigated. In [17], the optimal content caching probability that maximizes the hit probability is derived. The results are extended to heterogeneous cellular networks in [18] which shows that caching the most popular contents in the macro BSs is almost optimal while it is in general not optimal for SBSs.

3) Caching in Heterogeneous Networks: Extensive works have been carried out to understand the performance gain of caching for heterogeneous networks (HetNets) and stochastic geometry is the commonly used approach. In [19], the optimal probabilistic caching to maximize the successful delivery probability is considered in a multi-tier HetNet. The cache-enabled heterogeneous signal-antenna cellular networks are investigated in [20]. The optimal probabilistic content placement for the interference-limited cases is derived, and the result shows that the optimal placement probability is linearly proportional to the square root of the content popularity with an offset depending on BS caching capabilities. Caching policies to maximization of success probability and area spectral efficiency of cache-enabled HetNets are studied in [21], and the results show that the optimal caching probability is less skewed to maximize the success probability but is more skewed to maximize the area spectral efficiency. The work of [22] proposes a joint BS caching and cooperation for maximizing the successful transmission probability in a multitier HetNet. A local optimum is obtained in the general case and global optimal solutions are achieved in some special cases. Cache-based channel selection diversity and network interference are studied in [23] in stochastic wireless caching helper networks, and solutions for noise-limited networks and interference-limited networks are derived, respectively.

\section{B. Contributions and Organization}

The existing caching design for SBSs are restricted to the single-antenna case and mainly for the $\mu$-wave band. Little is known about the impact of multiple antennas at the densely deployed SBSs and the adoption of mm-wave band on the successful content delivery and the optimal content placement. Analyzing multi-antenna networks using stochastic geometry is a known difficulty, as acknowledged in [24]. In contrast to existing works, in this paper we analyze the performance of caching in multi-antenna SBSs in $\mu$-wave and mm-wave networks, and propose probabilistic content placement schemes to maximize the performance of content delivery. The main contributions of this paper are summarised as follows:

- Derivation of successful content delivery probability (SCDP) of multi-antenna SBSs. We use stochastic geometry to model wireless caching in multi-antenna dense small cell networks in both $\mu$-wave and mm-wave bands. The SCDPs for both types of cache-enabled SBSs are derived. The results characterize the dependence of the SCDPs on parameters such as channel effects, caching placement probability, SBS density, transmission power and number of antennas.

- Development of a near-optimal cross-entropy optimization (CEO) method for a general distribution of content requests. The derived SCDPs do not admit a closed form, and are highly complex to optimize. To tackle this difficulty, we first propose a constrained CEO (CCEO) based algorithm that optimizes the SCDPs. The original unconstrained CEO algorithm is a stochastic optimization method based on adaptive importance sampling that can achieve the near-optimal solution with moderate complexity and guaranteed convergence [25]. We adapt this method to deal with the caching capacity constraints and the probabilities constraints in our problem.

- Design of a simple heuristic content placement algorithm. To further reduce the complexity, we propose a heuristic two-stage algorithm to maximize the SCDP via probabilistic content placement when the content request probability follows the Zipf distribution [26]. The algorithm is designed by combining MPC and caching diversity (CD) schemes while taking into account the content popularity. The solution demonstrates near-optimal performance in single-antenna systems, and various advantages in multiantenna scenarios.

- Numerical results show that in contrast to the traditional way of deploying much higher density SBSs or installing many more antennas, increasing caching capacity at mm-wave SBSs provides a low-cost solution to achieve comparable SCDP performance as $\mu$-wave systems.

The rest of this paper is organized as follows. The system model is presented in Section II. The analysis of SCDPs for $\mu$-wave and mm-wave systems are provided in Section III. Two probabilistic content placement schemes are described in Section IV. Simulation and numerical results as well as discussions are given in Section $\mathrm{V}$, followed by concluding remarks in Section VI.

\section{SySTEM MODEL}

We consider a cache-enabled dense small cell networks consisting of the $\mu$-wave and mm-wave SBSs tiers. In such 


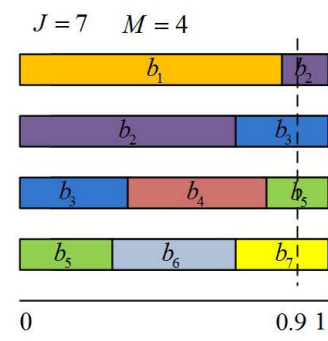

Fig. 1. Probabilistic content placement strategy.

networks, each user equipment (UE) in a tier is associated with the nearest SBS that has cached the desired content, and the optimal designs of content placement under such association assumption can address the concern that operators are required to place the content caches close to UEs [27]. We assume that there is a finite content library denoted as $\mathcal{F}:=\left\{f_{1}, \ldots, f_{j}, \ldots, f_{J}\right\}$, where $f_{j}$ is the $j$-th most popular content and the number of contents is $J$, we assume each content has normalized size of 1 and each BS can only store up to $M$ contents [16], [20], [23]. The analysis and optimization can be applied to the case of unequal content sizes. It is assumed that $M \ll J$. The request probability for the $j$-th content is $a_{j}$, and $\sum_{j=1}^{J} a_{j}=1$. Without loss of generality, we assume the contents are sorted according to a descending order of $a_{j}$.

\section{A. Probabilistic Content Placement}

We consider a probabilistic caching model where the content is independently stored with the same probability in all SBSs of the same tier (either $\mu$-wave or mm-wave) [17]. Let $b_{j}$ denote the probability that the $j$-th content is cached at a SBS. Fig. 1 shows an example of probabilistic caching with $J=7$ and $M=4$, where the contents $\left\{f_{2}, f_{3}, f_{5}, f_{7}\right\}$ are cached at a SBS by drawing uniformly a random number which is 0.9 in this example. In the probabilistic caching strategy, the caching probability $\boldsymbol{b}=\left\{b_{1}, \ldots b_{j}, \ldots b_{J}\right\}$ needs to satisfy the following conditions:

$$
\begin{aligned}
\sum_{j=1}^{J} b_{j} & \leq M \\
0 & \leq b_{j} \leq 1, \quad \forall j .
\end{aligned}
$$

Note that although the probabilistic caching strategy is used, implementation of it will allow each SBS to always cache the maximum amount of total contents up to its caching capacity $M$.

\section{B. Downlink Transmission}

In the considered downlink networks, each $\mu$-wave SBS is equipped with $N_{\mu}$ antennas, and each mm-wave SBS has directional mm-wave antennas. All UEs are single-antenna nodes, in the both $\mu$-wave and mm-wave, only one singleantenna user is allowed to communicate with the SBS at one time slot. ${ }^{2}$ The positions of $\mu$-wave SBSs are modeled

\footnotetext{
${ }^{2}$ In dense small cell networks, we assume that the density of users is much higher than the density of $\mu$-wave or mm-wave SBSs and this can be handled by using multiple access techniques [28].
}

by a homogeneous Poisson point process (HPPP) $\Phi^{\mu}$ with the density $\lambda_{\mu}$, and the positions of mm-wave SBSs are modeled by an independent HPPP $\Phi^{\mathrm{mm}}$ with the density $\lambda_{\mathrm{mm}}$. Define $\Phi_{j}^{\mu}$ and $\Phi_{j}^{\mathrm{mm}}$ as the point process corresponding to all SBSs that cache the content $j$ in the $\mu$-wave tier and the mm-wave tier with the density $b_{j} \lambda_{\mu}$ and $b_{j} \lambda_{\mathrm{mm}}$, respectively.

1) $\mu$-wave Tier: In the $\mu$-wave tier, the maximum-ratio transmission beamforming is adopted at each SBS. All channels undergo independent identically distributed (i.i.d.) quasistatic Rayleigh block fading. Without loss of generality, when a typical $\mu$-wave UE located at the origin $o$ requests the content $j$ from the associated $\mu$-wave BS $X_{o}$ that has cached this content, its received signal-to-interference-plus-noise ratio (SINR) is given by

$$
\mathrm{SINR}_{j}^{\mu}=\frac{P_{\mu} h_{j}^{\mu} L\left(\left|X_{j}^{\mu}\right|\right)}{\mathcal{I}_{j}^{\mu}+\overline{\mathcal{I}}_{j}^{\mu}+\sigma_{\mu}^{2}},
$$

where $P_{\mu}$ is the transmit power, $h_{j}^{\mu} \sim \Gamma\left(N_{\mu}, 1\right)$ is the the equivalent small-scale fading channel power gain between the typical $\mu$-wave UE and its serving $\mu$-wave SBS, where $\Gamma\left(k_{1}, k_{2}\right)$ denotes Gamma distribution, with a shape parameter $k_{1}$ and a scale parameter $k_{2}$. The path loss is $L\left(\left|X_{j}^{\mu}\right|\right)=$ $\beta_{\mu}\left(\left|X_{j}^{\mu}\right|\right)^{-\alpha_{\mu}}$ with the distance $\left|X_{j}^{\mu}\right|$, where $\beta_{\mu}$ is the frequency dependent constant parameter and $\alpha_{\mu}$ is the path loss exponent. The $\sigma_{\mu}^{2}$ is the noise power at a $\mu$-wave UE. The inter-cell interference $\mathcal{I}_{j}^{\mu}$ and $\overline{\mathcal{I}}_{j}^{\mu}$ are given by

$$
\left\{\begin{array}{l}
\mathcal{I}_{j}^{\mu}=\sum_{i \in \Phi_{j}^{\mu} \backslash X_{o}} P_{\mu} h_{i, o} L\left(\left|X_{i, o}\right|\right), \\
\overline{\mathcal{I}}_{j}^{\mu}=\sum_{k \in \bar{\Phi}_{j}^{\mu}} P_{\mu} h_{k, o} L\left(\left|X_{k, o}\right|\right) .
\end{array}\right.
$$

In (3), $\Phi_{j}^{\mu} \backslash X_{o}$ is the point process with density $b_{j} \lambda_{\mu}$ corresponding to the interfering SBSs that cache the content $j$, and $\bar{\Phi}_{j}^{\mu}=\Phi^{\mu}-\Phi_{j}^{\mu}$ with density $\left(1-b_{j}\right) \lambda_{\mu}$ is the point process corresponding to the interfering SBSs that do not store the content $j$. The $h_{i, o}, h_{k, o} \sim \exp (1)$ are the interfering channel power gains that follow the exponential distribution, and $\left|X_{i, o}\right|,\left|X_{k, o}\right|$ denote the distances between the interfering SBSs and the typical UE.

2) mm-wave Tier: In the mm-wave tier, we assume that the directional beamforming is adopted at each mm-wave SBS and small-scale fading is neglected, since small-scale fading has little change in received power as verified by the practical mm-wave channel measurements in [29]. Note that the traditional small-scale fading distributions are invalid for mm-wave modeling due to mm-wave sparse scattering environment [30]. Unlike the conventional $\mu$-wave counterpart, mm-wave transmissions are highly sensitive to the blockage. According to the average line-of-sight (LOS) model in [31], [32], we consider that the mm-wave link is LOS if the communication distance is less than $D_{L}$, and otherwise it is none-line-of-sight (NLOS). Moreover, the existing literature has confirmed that mm-wave transmissions tend to be noise-limited and interference is weak [31], [33]. Therefore, when a typical mm-wave UE requests the content $j$ from the associated mm-wave SBS that 
has cached this content, its received SINR is given by

$$
\mathrm{SINR}_{j}^{\mathrm{mm}}=\frac{P_{\mathrm{mm}} G_{\mathrm{mm}} L\left(\left|Y_{j}^{\mathrm{mm}}\right|\right)}{\sigma_{\mathrm{mm}}^{2}},
$$

where $P_{\mathrm{mm}}$ is the transmit power of the mm-wave SBS, $G_{\mathrm{mm}}$ is the main-lobe gain of using direction beamforming and equal to number of antenna elements [34]. The path loss is expressed as $L\left(\left|Y_{j}^{\mathrm{mm}}\right|\right)=\beta_{\mathrm{mm}}\left(\left|Y_{j}^{\mathrm{mm}}\right|\right)^{-\alpha}$ with the distance $\left|Y_{j}^{\mathrm{mm}}\right|$ and frequency-dependent parameter $\beta_{\mathrm{mm}}$. The path loss exponent $\alpha=\alpha_{\mathrm{L}}$ when it is a LOS link and $\alpha=\alpha_{\mathrm{N}}$ when it is an NLOS link. The $\sigma_{\mathrm{mm}}^{2}$ is the combined power of noise and weak interference. ${ }^{3}$

\section{Successful Content Delivery Probability}

In this paper, SCDP is used as the performance indicator, which represents the probability that a content requested by a typical UE is both cached in the network and can be successfully transmitted to the UE. We assume that each content has $\eta$ bits, and the delivery time needs to be less than $T$. By using the Law of total probability, the SCDP in the $\mu$-wave tier is calculated as

$$
\begin{aligned}
\mathcal{P}_{\mathrm{SCD}}^{\mu} & =\sum_{j=1}^{J} a_{j} \operatorname{Pr}\left(W_{\mu} \log _{2}\left(1+\operatorname{SINR}_{j}^{\mu}\right) \geqslant \frac{\eta}{T}\right) \\
& =\sum_{j=1}^{J} a_{j} \operatorname{Pr}\left(\operatorname{SINR}_{j}^{\mu}>\varphi_{\mu}\right),
\end{aligned}
$$

where $W_{\mu}$ is the $\mu$-wave bandwidth allocated to a typical user (frequency-division multiple access (FDMA) is employed when multiple users are served by a SBS in this paper), and $\varphi_{\mu}=2^{\frac{\eta}{W_{\mu}^{T}}}-1$. Likewise, in the mm-wave tier, the SCDP is calculated as

$$
\begin{aligned}
\mathcal{P}_{\mathrm{SCD}}^{\mathrm{mm}} & =\sum_{j=1}^{J} a_{j} \operatorname{Pr}\left(W_{\mathrm{mm}} \log _{2}\left(1+\operatorname{SINR}_{j}^{\mathrm{mm}}\right) \geqslant \frac{\eta}{T}\right) \\
& =\sum_{j=1}^{J} a_{j} \operatorname{Pr}\left(\mathrm{SINR}_{j}^{\mathrm{mm}}>\varphi_{\mathrm{mm}}\right),
\end{aligned}
$$

where $W_{\mathrm{mm}}$ is the mm-wave bandwidth allocated to a typical user, and $\varphi_{\mathrm{mm}}=2^{\frac{\eta}{W_{\mathrm{mm} T}}}-1$. The rest of this section is devoted to deriving the SCDPs in (5) and (6).

\section{A. $\mu$-wave Tier}

Based on (2) and (5), the SCDP in the $\mu$-wave tier can be derived and summarized below.

Theorem 1: In the cache-enabled $\mu$-wave tier, the SCDP is given by

$$
\mathcal{P}_{\mathrm{SCD}}^{\mu}=\sum_{j=1}^{J} a_{j} \mathcal{P}_{j, \mathrm{SCD}}^{\mu}\left(b_{j}\right),
$$

\footnotetext{
${ }^{3} \mathrm{~mm}$-wave in dense networks works in the noise-limited regime, since the high path loss impairs the interference, which could improve the signal directivity [33]. In contrast to the sub-6 GHz counterpart which is usually interference-limited, mm-wave networks tend to be noise-limited when the BS density is not extremely dense, due to the narrow beam and blocking effects [35]. For completeness, we also incorporate weak interference here.
}

where $P_{j, \mathrm{SCD}}^{\mu}\left(b_{j}\right)$ denotes the probability that the $j$-th request content is successfully delivered to the $\mu$-wave UE by its serving SBS, and is expressed as

$$
P_{j, \mathrm{SCD}}^{\mu}\left(b_{j}\right)=\int_{0}^{\infty} P_{\mathrm{cov}}^{\mu}\left(x, b_{j}\right) f_{\left|X_{j}^{\mu}\right|}(x) d x,
$$

where $P_{\text {cov }}^{\mu}\left(x, b_{j}\right)$ is given by (10), (11) and (12) at the top of next page, which represents the conditional coverage probability that the received SINR is larger than $\varphi_{\mu}$ given a typical communication distance $x . f_{\left|X_{j}^{\mu}\right|}(x)$ is the probability density function (PDF) of the distance $\left|X_{j}^{\mu}\right|$ between a typical $\mu$-wave UE and its nearest serving SBS that stores content $j$, and is given by [36]

$$
f_{\left|X_{j}^{\mu}\right|}(x)=2 \pi b_{j} \lambda_{\mu} x e^{-\pi b_{j} \lambda_{\mu} x^{2}} .
$$

Proof 1: Please see Appendix A.

Note that $P_{j, \mathrm{SCD}}^{\mu}\left(b_{j}\right)$ becomes the probability of successful transmission from the serving SBS to the typical user when $b_{j}=1$ in traditional $\mu$-wave networks without caching. We see that the SCDP expression for multi-antenna systems is much complicated, compared to the closed-form expression for single-antenna systems in [20].

\section{B. mm-wave Tier}

Based on (4) and (6), the SCDP in the mm-wave tier can be derived and summarized below.

Theorem 2: In the cache-enabled mm-wave tier, the SCDP is given by

$$
\mathcal{P}_{\mathrm{SCD}}^{\mathrm{mm}}=\sum_{j=1}^{J} a_{j} \mathcal{P}_{j, \mathrm{SCD}}^{\mathrm{mm}, \mathrm{L}}\left(b_{j}\right)+\sum_{j=1}^{J} a_{j} \mathcal{P}_{j, \mathrm{SCD}}^{\mathrm{mm}, \mathrm{N}}\left(b_{j}\right),
$$

where $\mathcal{P}_{j, \mathrm{SCD}}^{\mathrm{mm}, \mathrm{L}}\left(b_{j}\right)$ and $\mathcal{P}_{j, \mathrm{SCD}}^{\mathrm{mm}, \mathrm{N}}\left(b_{j}\right)$ denote that probabilities that the content $j$ is successfully delivered when the mm-wave UE is connected to its serving mm-wave SBS via LOS link and NLOS link, and are given by

$$
\mathcal{P}_{j, \mathrm{SCD}}^{\mathrm{mm}, \mathrm{L}}\left(b_{j}\right)=1-e^{-\left(\min \left(D_{\mathrm{L}}, d_{\mathrm{L}}\right)\right)^{2} \pi b_{j} \lambda_{\mathrm{mm}}},
$$

and

$$
\mathcal{P}_{j, \mathrm{SCD}}^{\mathrm{mm}, \mathrm{N}}\left(b_{j}\right)=e^{-D_{\mathrm{L}}^{2} \pi b_{j} \lambda_{\mathrm{mm}}}-e^{-\left(\max \left(D_{\mathrm{L}}, d_{\mathrm{N}}\right)\right)^{2} \pi b_{j} \lambda_{\mathrm{mm}}},
$$

respectively, where $d_{\mathrm{L}}=\left(\frac{P_{\mathrm{mm}} G_{\mathrm{mm}} \beta_{\mathrm{mm}}}{\varphi_{\mathrm{mm}} \sigma_{\mathrm{mm}}^{2}}\right)^{\frac{1}{\alpha_{\mathrm{L}}}}$ and $d_{\mathrm{N}}=$ $\left(\frac{P_{\mathrm{mm}} G_{\mathrm{mm}} \beta_{\mathrm{mm}}}{\varphi_{\mathrm{mm}} \sigma_{\mathrm{mm}}^{2}}\right)^{\frac{1}{\alpha_{\mathrm{N}}}}$.

Proof 2: Please see Appendix B.

\section{Optimization OF Probabilistic CONTENT Placement}

In this section, we aim to maximize the SCDP by optimizing the probabilistic content placement $\left\{b_{j}\right\}$. The main difficulty is that the SCDP expressions (7) and (13) do not have a closed form for the multi-antenna case and whether they are concave with regard to $\left\{b_{j}\right\}$ is unknown, which is much more challenging than the single-antenna SBS case studied in [20]. Therefore, the optimal content placement problem for the multi-antenna case is distinct. To tackle this new problem, here 


$$
\begin{aligned}
P_{\mathrm{cov}}^{\mu}\left(x, b_{j}\right)=\sum_{n=0}^{N_{\mu}-1} \frac{\left(x^{\alpha_{\mu}}\right)^{n}}{n !(-1)^{n}} \sum_{\left\{t_{q}\right\}_{q=1}^{n} \in \Theta} \frac{n !}{\prod_{q=1}^{n} t_{q} !(q !)^{t_{q}}} \exp \left(-\frac{\varphi_{\mu} \sigma_{\mu}^{2} x^{\alpha_{\mu}}}{P_{\mu} \beta_{\mu}}-2 \pi b_{j} \lambda_{\mu} \frac{\varphi_{\mu} x^{2}}{\alpha_{\mu}-2}\right. \\
\left.\quad \times{ }_{2} F_{1}\left[1, \frac{-2+\alpha_{\mu}}{\alpha_{\mu}}, 2-\frac{2}{\alpha_{\mu}},-\varphi_{\mu}\right]-\frac{2 \pi^{2}}{\alpha_{\mu}}\left(1-b_{j}\right) \lambda_{\mu}\left(\varphi_{\mu} x^{\alpha_{\mu}}\right)^{\frac{2}{\alpha_{\mu}}} \csc \left(\frac{2 \pi}{\alpha_{\mu}}\right)\right) \prod_{q=1}^{n}\left(\mathcal{T}^{(q)}\left(x^{\alpha_{\mu}}\right)\right)^{t_{q}},
\end{aligned}
$$

where $\Theta \triangleq\left\{\left\{t_{q}\right\}_{q=1}^{n} \mid \sum_{q=1}^{n} q \cdot t_{q}=n, t_{q}\right.$ is an integer, $\left.\forall n\right\}, \csc (\cdot)$ is the Cosecant trigonometry function, and

$$
\begin{aligned}
\mathcal{T}_{j}^{(1)}\left(x^{\alpha_{\mu}}\right)= & -\frac{\varphi_{\mu} \sigma_{\mu}^{2}}{P_{\mu} \beta}-2 \pi b_{j} \lambda_{\mu} x^{2-\alpha_{\mu}} \varphi_{\mu} \frac{\alpha_{\mu}-2+2\left(1+\varphi_{\mu}\right)_{2} F_{1}\left[1, \frac{\alpha_{\mu}-2}{\alpha_{\mu}}, 2-\frac{2}{\alpha_{\mu}},-\varphi_{\mu}\right]}{\left(1+\varphi_{\mu}\right)\left(\alpha_{\mu}-1\right) \alpha_{\mu}} \\
& -4 \pi^{2}\left(1-b_{j}\right) \lambda_{\mu}\left(\varphi_{\mu}^{\alpha_{\mu}} x\right)^{2-\alpha_{\mu}} \csc \left(\frac{2 \pi}{\alpha_{\mu}}\right), \\
\mathcal{T}_{j}^{(q)}\left(x^{\alpha_{\mu}}\right)= & 2 \pi b_{j} \lambda_{\mu} q !(-1)^{q} x^{-\left(2+\alpha_{\mu}\right)(1+q)} \varphi_{\mu}^{-q(1+q)} \frac{{ }_{2} F_{1}\left[1+q, \frac{2+\alpha_{\mu}}{\alpha_{\mu}}, 2+\frac{2}{\alpha_{\mu}},-\frac{1}{\varphi_{\mu}}\right]}{2+\alpha_{\mu}}+2 \pi\left(1-b_{j}\right) \lambda_{\mu} q !(-1)^{q} \\
& \times\left(x^{\alpha_{\mu}}\right)^{-q+\frac{2}{\alpha_{\mu}}} \varphi_{\mu}^{\frac{2}{\alpha_{\mu}}} \frac{\Gamma\left(q-\frac{2}{\alpha_{\mu}}\right) \Gamma\left(\frac{2+\alpha_{\mu}}{\alpha_{\mu}}\right)}{\alpha_{\mu} \Gamma(1+q)}, q>1 .
\end{aligned}
$$

we propose two algorithms, the first one is developed based on the CEO method that can achieve near-optimal performance, and the other two-stair scheme is based on the combination of MPC and CD content placement schemes with reduced complexity.

\section{A. The Near-Optimal CCEO Algorithm}

The optimal caching placement probability in the multiantenna case is hard to achieve, so we introduce CEO to resolve the difficulty of maximizing the SCDP by optimizing the probabilistic content placement. CEO is an adaptive variance algorithm for estimating probabilities of rare events. The rationale of the CEO algorithm is to first associate with each optimization problem a rare event estimation problem, and then to tackle this estimation problem efficiently by an adaptive algorithm. The outcome of this algorithm is the construction of a random sequence of solutions which converges probabilistically to the optimal or near-optimal solution [25], [37]. The CEO method involves two iterative steps. The first one is to generate samples of random data according to a specified random (normally Gaussian) distribution. And the second step updates the parameters of the random distribution, based on the sample data to produce better samples in the next iteration. The CEO algorithm has been successfully applied to a wide range of difficult optimization tasks such as traveling salesman problem and antenna selection problem in multi-antenna communications [38]. It has shown superior performance in solving complex optimization problems compared to commonly used simulated annealing (SA) and genetic algorithm (GA) [39] that are based on random search.

The original principle of the CEO algorithm was proposed for unconstrained optimization. To deal with the constraints on the probabilities $\left\{b_{j}\right\}$ and the content capacity constraint, we propose a CCEO algorithm as shown in Algorithm 1. In the proposed CCEO algorithm, we force the randomly generated samples to be within the feasible set $\left\{b_{j} \mid 0 \leq b_{j} \leq 1, \forall j\right\}$ in the Project step. To satisfy the constraint of $\sum_{j}^{J} b_{j} \leqslant M$, we introduce a penalty function $H\left(\sum_{j}^{J} b_{j}-M\right)$ to the original objective function in the Modification step, where $H$ is a large positive number that represents the parameter for the penalty function. The dynamic Smoothing step will prevent the result from converging to a sub-optimal solution. It can be seen that at each iteration, the main computation is to evaluate the objective functions for $N_{s}$ times and no gradient needs to be calculated, so the complexity is moderate and can be further controlled to achieve a complexity-convergence tradeoff.

In Fig. 2, we provide an example of the iterative results of content placement probabilities with iteration indices $t=1$, $t=5, t=20$, and $t=70$. In this example, the algorithm converges when $t=70$. Each sub-figure presents the resulting mean value of $\boldsymbol{\mu}_{t}$ at the end of iteration $t$, and it will help to generate random samples in next iteration. We can observe that when $t=20$, the caching placement probability is quite close to the converged solution, which could significantly reduce the complexity. Overall the CEO algorithm converges fast and is an efficient method to find the near-optimal SCDP result, and the complexity of the CEO algorithm is $\mathcal{O}\left(n^{3}\right)$ [40]. It is also noted that the top ranked contents are cached with probability $b_{j}=1$, while to make effective use of the rest caching space, caching diversity is more important. Based on this observation, we design a low-complexity heuristic scheme in the next subsections.

\section{B. Two-Stair Scheme for the $\mu$-wave Tier}

To further reduce the complexity of the optimization, we devise a simple two-stair (TS) scheme, when the content 


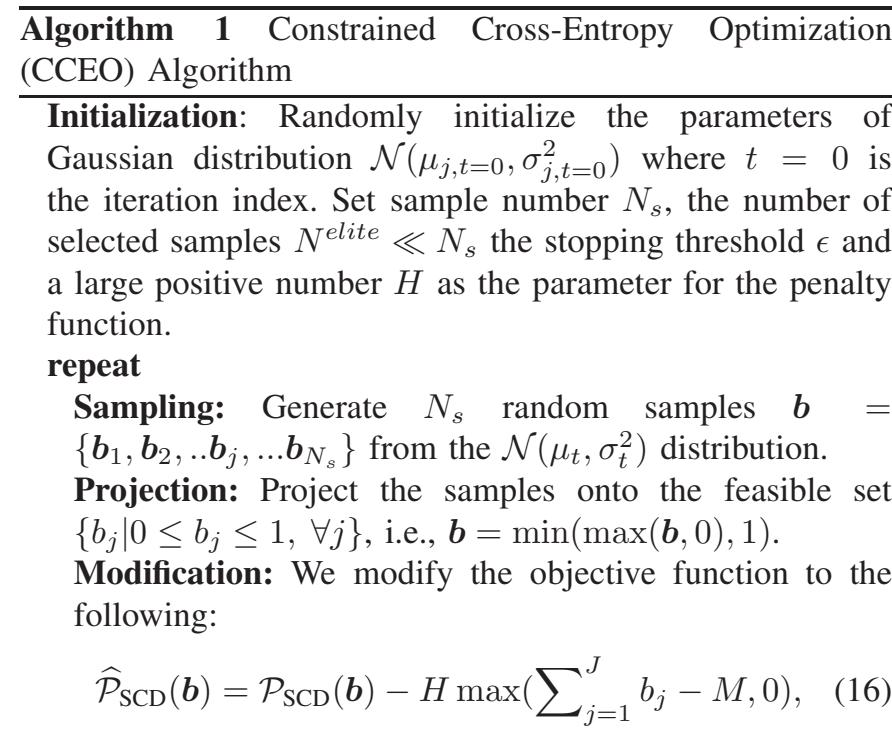

where $\mathcal{P}_{\mathrm{SCD}}(\boldsymbol{b})$ is the original objective function in (7) and (13) for $\mu$-wave and mm-wave, respectively.

Selection: Evaluate $\widehat{\mathcal{P}}_{\mathrm{SCD}}(\boldsymbol{b})$ for $N_{s}$ samples $\boldsymbol{b}$. Let $\mathcal{I}$ be the indices of the $N^{\text {elite }}$ selected best performing samples with $\widehat{\mathcal{P}}_{\mathrm{SCD}}(\boldsymbol{b})$.

Updating: for all $j \in \mathcal{F}$, calculate the sampling mean and variance:

$$
\begin{aligned}
& \widetilde{\mu}_{i j}=\sum_{i \in \mathcal{I}} b_{i j} / N^{\text {elite }} \\
& \tilde{\sigma}_{i j}^{2}=\sum_{i \in \mathcal{I}}\left(b_{i j}-\widetilde{\mu}_{i j}\right)^{2} / N^{\text {elite }} .
\end{aligned}
$$

Smoothing: The Gaussian distribution parameters are updated as follows,

$$
\begin{aligned}
\boldsymbol{\mu}_{t} & =\iota \widetilde{\boldsymbol{\mu}}_{t}+(1-\iota) \boldsymbol{\mu}_{t-1}, \\
\boldsymbol{\sigma}_{t}^{2} & =\beta_{t} \widetilde{\boldsymbol{\sigma}}_{t}^{2}+\left(1-\beta_{t}\right) \boldsymbol{\sigma}_{t-1}^{2} .
\end{aligned}
$$

In particular, $\alpha$ is a fixed smoothing parameter $(0.5 \leqslant \alpha \leqslant$ $0.9)$ while $\beta_{t}$ is a dynamic smoothing parameter given by

$$
\beta_{t}=\beta-\beta\left(1-\frac{1}{t}\right)^{q},
$$

where $\beta$ is a fixed smoothing parameter $(0.8 \leqslant \beta \leqslant 0.99)$, and $q$ is an integer with a typical value between 5 and 10 . Increment: $t=t+1$.

until A convergence criterion is satisfied, e.g., $\max _{j \in \mathcal{F}}\left(\boldsymbol{\sigma}_{t}^{2}\right)<\epsilon$ Output: The optimal caching probability is $\boldsymbol{b}^{\stackrel{\jmath}{\in}=\boldsymbol{F}_{t}}$.

popularity is modeled as the Zipf distribution [14], [17], [26] based on empirical studies, which is given by

$$
a_{j}=j^{-\gamma} / \sum_{m=1}^{J} m^{-\gamma}
$$

where $\gamma$ is the Zipf exponent that represents the popularity skewness.

In the TS scheme, a fraction of caching space $\varepsilon M(0 \leq$ $\varepsilon \leq 1$ ) at a SBS is allocated to store the most popular contents which is called the MPC region. The remaining cache space is allocated to randomly store the contents with
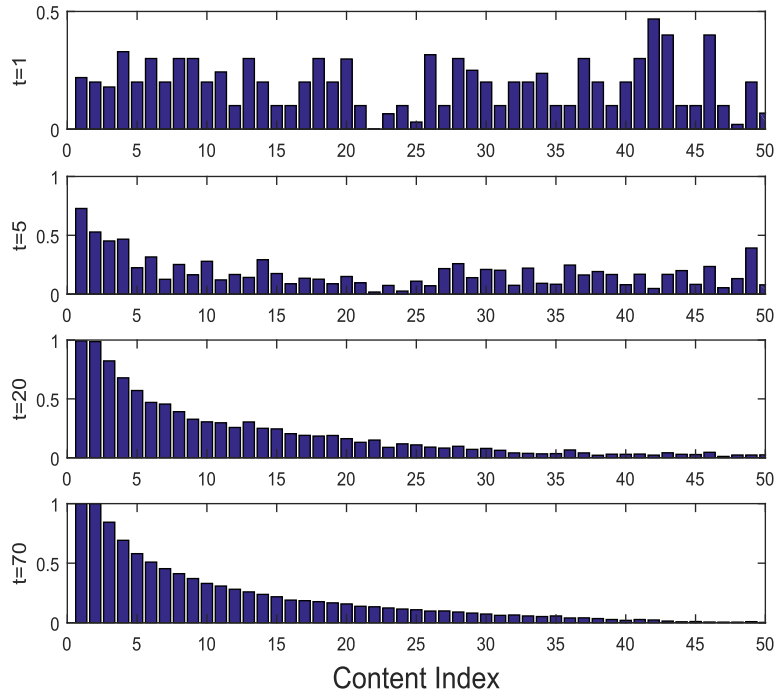

Fig. 2. Evolution of the content placement probabilities in the CCEO algorithm with parameters $\gamma=1.5, J=50, M=10$.



Fig. 3. Two-Stair probabilistic content placement strategies.

certain probabilities and is called the $\mathrm{CD}$ region. As illustrated in Fig. 3, in the 'Two-Stair' caching scheme, the contents in the $\mathrm{CD}$ region are cached with a common probability $\varpi$. The rest of the contents are not cached and must be fetched through the backhaul links. These content placement schemes will be studied in detail in the rest of this section.

In this scheme, the content placement probabilities $\left\{b_{j}\right\}$ need to satisfy the following conditions:

$$
\left\{\begin{array}{l}
b_{1}=\ldots=b_{\lfloor\varepsilon M\rfloor}=1, \\
b_{\lfloor\varepsilon M\rfloor+1}=\ldots=b_{\lfloor\varepsilon M\rfloor+\left\lfloor\frac{M-\lfloor\varepsilon M\rfloor}{\varpi}\right\rfloor}=\varpi, \\
b_{\lfloor\varepsilon M\rfloor+\left\lfloor\frac{M-\lfloor\varepsilon M\rfloor}{\varpi}\right\rfloor+1}=\ldots=b_{J}=0,
\end{array}\right.
$$

which are characterized by two variables $\varepsilon$ and $\varpi$, where $\varpi$ denotes the common probability value that content $j$ in the $\mathrm{CD}$ region is stored at a SBS.

As such, the $\mu$-wave SCDP (7) can be expressed as

$$
\mathcal{P}_{\mathrm{SCD}}^{\mu}=\sum_{j=1}^{\lfloor\varepsilon M\rfloor} a_{j} \mathcal{P}_{j, \mathrm{SCD}}^{\mu}(1)+\sum_{j=\lfloor\varepsilon M\rfloor+1}^{\lfloor\varepsilon M\rfloor+\left\lfloor\frac{\lfloor-\lfloor\varepsilon M\rfloor}{\varpi}\right\rfloor} a_{j} \mathcal{P}_{j, \mathrm{SCD}}^{\mu}(\varpi) .
$$

It is seen in (24) that contents $\{1, \cdots,\lfloor\varepsilon M\rfloor\}$ have the same $\operatorname{SCDP} \mathcal{P}_{j, \mathrm{SCD}}^{\mu}(1)$, and contents $\{\lfloor\varepsilon M\rfloor+1, \cdots$, $\left.\lfloor\varepsilon M\rfloor+\left\lfloor\frac{M-\lfloor\varepsilon M\rfloor}{\varpi}\right\rfloor\right\}$ have the same $\operatorname{SCDP} \mathcal{P}_{j, \mathrm{SCD}}^{\mu}(\varpi)$. Our aim is to maximize the overall SCDP, and the problem is 
formulated as

$$
\begin{aligned}
& \max _{\varepsilon, \varpi} \mathcal{P}_{\mathrm{SCD}}^{\mu} \text { in }(24) \\
& \text { s.t. } \mathrm{C} 1: 0 \leq \varepsilon \leq 1, \\
& \text { s.t. } \mathrm{C} 2: 0 \leq \varpi \leq 1, \\
& \text { s.t. } \mathrm{C} 3: \mathbf{1}(\varepsilon=1) \varpi=0,
\end{aligned}
$$

where $\mathbf{1}(A)$ is the indicator function that returns one if the condition $\mathrm{A}$ is satisfied. The convexity of the problem (25) is unknown, and finding its global optimal solution is challenging. To obtain an efficient caching placement solution, we first use the following approximations [41]

$$
\begin{aligned}
\sum_{j=1}^{\lfloor\varepsilon M\rfloor} a_{j} & \approx \frac{(\varepsilon M)^{1-\gamma}-1}{J^{1-\gamma}-1}, \\
\sum_{j=\lfloor\varepsilon M\rfloor+1}^{\lfloor\varepsilon M\rfloor+\left\lfloor\frac{M-\lfloor\varepsilon M\rfloor}{\varpi}\right\rfloor} a_{j} & \approx \frac{\left(\varepsilon M+\frac{M(1-\varepsilon)}{\varpi}\right)^{1-\gamma}-1}{J^{1-\gamma}-1}-\frac{(\varepsilon M)^{1-\gamma}-1}{J^{1-\gamma}-1} \\
& =\frac{M^{1-\gamma}}{J^{1-\gamma}-1}\left[\left(\varepsilon+\frac{(1-\varepsilon)}{\varpi}\right)^{1-\gamma}-\varepsilon^{1-\gamma}\right],
\end{aligned}
$$

respectively, based on the fact that for Zipf popularity with $0<\gamma, \gamma \neq 1$ and $M \ll J$, we have $\sum_{j=1}^{M} j^{-\gamma} / \sum_{m=1}^{J} m^{-\gamma} \approx\left(M^{1-\gamma}-1\right) /\left(J^{1-\gamma}-1\right)[41]$. Therefore, the objective function of (24) can be approximated as

$$
\begin{aligned}
\widetilde{\mathcal{P}}_{\mathrm{SCD}}^{\mu} \approx & \mathcal{P}_{j, \mathrm{SCD}}^{\mu}(1) \frac{M^{1-\gamma}}{J^{1-\gamma}-1} \varepsilon^{1-\gamma}-\frac{\mathcal{P}_{j, \mathrm{SCD}}^{\mu}(1)}{J^{1-\gamma}-1} \\
& +\mathcal{P}_{j, \mathrm{SCD}}^{\mu}(\varpi) \frac{M^{1-\gamma}}{J^{1-\gamma}-1}\left[\left(\varepsilon+\frac{(1-\varepsilon)}{\varpi}\right)^{1-\gamma}-\varepsilon^{1-\gamma}\right] .
\end{aligned}
$$

Note that for the special case of MPC caching, i.e., $\varepsilon=1, \varpi=0$, the above reduces to $\widetilde{\mathcal{P}}_{\mathrm{SCD}}^{\mu} \approx$ $\mathcal{P}_{j, \mathrm{SCD}}^{\mu}(1) \frac{M^{1-\gamma}-1}{J^{1-\gamma}-1}$.

Then the problem (25) can be approximated as

$$
\begin{aligned}
& \max _{\varepsilon, \varpi} \widetilde{\mathcal{P}}_{\mathrm{SCD}}^{\mu} \\
& \text { s.t. } \mathrm{C} 1-\mathrm{C} 3 .
\end{aligned}
$$

Because $\varepsilon$ and $\varpi$ are coupled in the objective function of (29), we use a decomposition approach to solve this problem. Since $M^{1-\gamma}$ is always positive, given $\varpi$, the optimal $\varepsilon$ is obtained by solving the following equivalent sub-problem:

$\max _{0 \leq \varepsilon \leq 1} \frac{1}{J^{1-\gamma}-1}\left[\left(\ell_{o}^{\mu}-1\right) \varepsilon^{1-\gamma}+\left(\varepsilon+\frac{(1-\varepsilon)}{\varpi}\right)^{1-\gamma}-\ell_{o}^{\mu}\right]$

where $\ell_{o}^{\mu}=\frac{\mathcal{P}_{j, \mathrm{SCD}}^{\mu}(1)}{\mathcal{P}_{j, \mathrm{SCD}}^{\mu}(\varpi)} \geqslant 1$ is independent of $\varepsilon$. Thus, we have the following theorem:

Theorem 3: The optimal solution of the problem (30) is given by

$$
\varepsilon^{*}=\min \left(\max \left(\varepsilon_{o}, 0\right), 1\right),
$$

where $\varepsilon_{o}=\left(\left(\left(\frac{\ell_{o}^{\mu}-1}{\varpi^{-1}-1}\right)^{-1 / \gamma}-1\right) \varpi+1\right)^{-1}$.
Proof 3: Please see Appendix C. For $\varepsilon_{o}$ to be in the range of $[0,1], \varpi$ should satisfy $0 \leqslant \varpi \leqslant \frac{1}{\ell_{o}^{\mu}}$.

Consequently, the problem (29) reduces to the following optimization problem about $\varpi$ only:

$$
\max _{\varepsilon=\varepsilon_{o}(\varpi), 0 \leqslant \varpi \leqslant \frac{1}{\ell_{o}^{\mu}}} \widetilde{\mathcal{P}}_{\mathrm{SCD}}^{\mu} .
$$

Since the problem (32) is non-convex, we propose to use Newton's method to solve it, which is shown in the Appendix D. Note that the Newton's method converges faster than the Karush-Kuhn-Tucker (KKT) method and the gradient-based method [42]. Suppose the obtained solution is $\hat{\varpi}$, then the optimal $\hat{\varpi}^{*}$ is $\min (\max (\hat{\varpi}, 0), 1)$, and the optimal $\varepsilon^{*}$ can be obtained from (31).

\section{Two-Stair Scheme for the mm-wave Tier}

Similar to the $\mu$-wave case, the SCDP of the mm-wave tier can be approximated by

$$
\begin{aligned}
\mathcal{P}_{\mathrm{SCD}}^{\mathrm{mm}} \approx & \left(\mathcal{P}_{j, \mathrm{SCD}}^{\mathrm{mm}, \mathrm{L}}(1)+\mathcal{P}_{j, \mathrm{SCD}}^{\mathrm{mm}, \mathrm{N}}(1)\right) \frac{(\varepsilon M)^{1-\gamma}-1}{J^{1-\gamma}-1} \\
& +\left(\mathcal{P}_{j, \mathrm{SCD}}^{\mathrm{mm}, \mathrm{L}}(\varpi)+\mathcal{P}_{j, \mathrm{SCD}}^{\mathrm{mm}, \mathrm{N}}(\varpi)\right) \\
& \times \frac{M^{1-\gamma}}{J^{1-\gamma}-1}\left[\left(\varepsilon+\frac{(1-\varepsilon)}{\varpi}\right)^{1-\gamma}-\varepsilon^{1-\gamma}\right] .
\end{aligned}
$$

Then the optimal two-stair content caching can be found obtained by solving the following problem:

$$
\begin{aligned}
& \max _{\varepsilon, \varpi} \mathcal{P}_{\mathrm{SCD}}^{\operatorname{mm}} \text { in }(33) \\
& \text { s.t. } \mathrm{C} 1-\mathrm{C} 3 .
\end{aligned}
$$

The problem (34) can be efficiently solved by following the decomposition approach. Given $\varpi$, the optimal $\varepsilon$ is obtained by solving the following equivalent sub-problem:

$$
\max _{0 \leq \varepsilon \leq 1} \frac{\left(\ell_{o}^{\mathrm{mm}}-1\right) \varepsilon^{1-\gamma}+\left(\varepsilon+\frac{(1-\varepsilon)}{\varpi}\right)^{1-\gamma}-\ell_{o}^{\mathrm{mm}}}{J^{1-\gamma}-1},
$$

where $\ell_{o}^{\mathrm{mm}}=\frac{\left(\mathcal{P}_{j, \mathrm{SCD}}^{\mathrm{mm}, \mathrm{L}}(1)+\mathcal{P}_{j, \mathrm{SCD}}^{\mathrm{mm}, \mathrm{N}}(1)\right)}{\left(\mathcal{P}_{j, \mathrm{SCD}}^{\mathrm{mm}, \mathrm{L}}(\varpi)+\mathcal{P}_{j, \mathrm{SCD}}^{\mathrm{mm}, \mathrm{N}}(\varpi)\right)}$. The rest procedures follow the same approach in the section IV-A, except that the derivation of the search direction to solve the optimal $\varpi^{*}$, which is provided Appendix E.

\section{RESUlTS AND Discussions}

In this section, the performance of the proposed caching schemes are evaluated by presenting numerical results. Performance comparison between cache-enabled $\mu$-wave and mm-wave systems is also highlighted. The system parameters are shown in Table I, unless otherwise specified. $1 \mathrm{GHz}$ and $60 \mathrm{GHz}$ are chosen for the $\mu$-wave and mm-wave frequency bands, respectively.

Fig. 4 verifies the SCDPs for content $j$ derived in Theorem 1 and Theorem 2 against the content placement probability. The analytical results are obtained from (8), (14) and (15). The SCDP for an arbitrary content $j$ is observed to be a monotonically increasing and concave function of the caching 
TABLE I

PARAMETER VAlues

\begin{tabular}{c|c}
\hline Parameters & Values \\
\hline \hline Number of Antenna in $\mu$-wave-SBS $\left(N_{\mu}\right)$ & 2 \\
\hline Main-lobe Array Gain in mm-wave-SBS $\left(G_{\mathrm{mm}}\right)$ & 2 \\
\hline LOS region $\left(D_{\mathrm{L}}\right)$ & $15 \mathrm{~m}$ \\
\hline Transmit power of each $\mu$-wave-SBS $P_{\mu}$ & $20 \mathrm{dBm}$ \\
\hline Transmit power of each mm-wave-SBS $P_{\mathrm{mm}}$ & $20 \mathrm{dBm}$ \\
\hline SBS's density for $\mu$-wave and mm-wave & $\lambda_{\mu}, \lambda_{\mathrm{mm}}=600 / \mathrm{km}^{2}$ \\
\hline Path loss exponent $f_{c}=1 \mathrm{GHz}$ & $\alpha_{\mu}=2.5$ \\
\hline Path loss exponent $f_{c}=60 \mathrm{GHz}[43]$ & $\alpha_{\mathrm{L}}=2.25, \alpha_{\mathrm{N}}=3.76$ \\
\hline Bit rate of each content $(\eta / T)$ & $4 \times 10^{5} \mathrm{bit} / \mathrm{s}$ \\
\hline Available bandwidth in $\mu$-wave $\left(W_{\mu}\right)$ & $10 \mathrm{MHz}$ \\
\hline Available bandwidth in mm-wave $\left(W_{\mathrm{mm}}\right)$ & $1 \mathrm{GHz}$ \\
\hline SBS cache capacity $(M)$ & 10 \\
\hline Content library size $(J)$ & 100 \\
\hline Zipf exponent $(\gamma)$ & $0 \sim 2$ \\
\hline
\end{tabular}

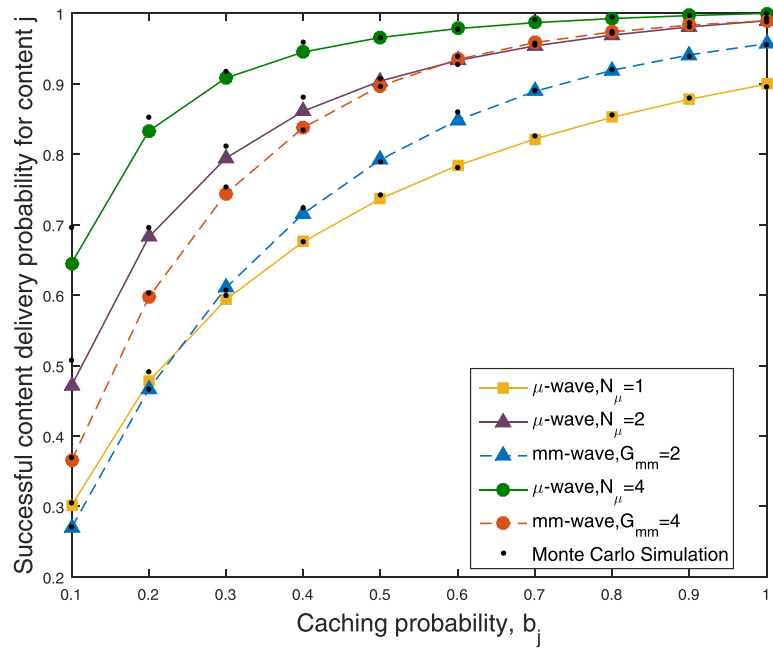

Fig. 4. Successful content delivery probability for content $j$ versus the content placement probability.

placement probability for both $\mu$-wave and mm-wave systems. Notice that all our derived analytical results match very well with those ones via Monte Carlo simulations averaged over 2,000 random user drops and marked by ' $'$ '.

In Fig. 5, we examine the comparison of successful transmission probabilities of $\mu$-wave from (8) and mm-wave from (14) and (15) as bit rate of each content varies, which corresponds to the case with caching placement probability $b_{j}=1$. It is seen that when content size is small, the $\mu$-wave system shows better performance than mm-wave, but as the content size increases, the mm-wave system outperforms the $\mu$-wave system for its ability to provide high capacity. The successful mm-wave transmission probability shows a 'ladder drop' effect, and this is because the mm-wave system combines LOS part and NLOS part. The LOS effect is limited to the region within the distance $D_{\mathrm{L}}$ ) while NLOS has a much wider coverage, so when the required content size is small, the performance is dominated by the NLOS part. However, the NLOS part cannot provide high capacity due to the much larger path loss exponent $\alpha_{\mathrm{N}}$, so its performance drops steeply as the bit rate of each content increases.



Fig. 5. Successful transmission probability, $\lambda_{\mu, \mathrm{mm}}=400 / \mathrm{km}^{2}, b_{j}=1$.
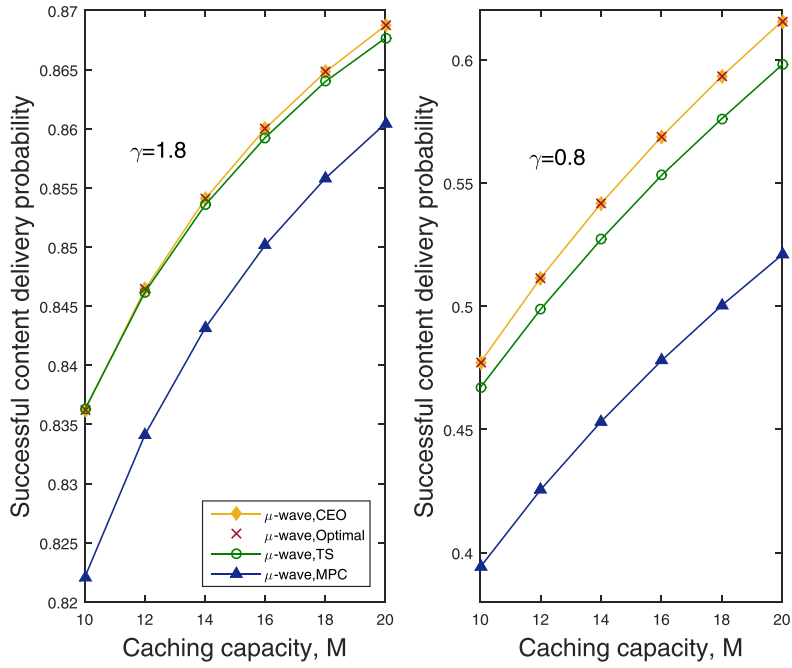

Fig. 6. Successful content delivery probability for $\mu$-wave single antennas.

Next, in Figs. 6-7, we compare the performance of the two proposed content placement schemes with the close-form optimal solution [20] and the intuitive MPC scheme [21] in the $\mu$-wave single-antenna case. Note that in the general multiantenna setting, the close-form optimal content placement is still unknown. The SCDP with different caching capacity $M$ is shown in Fig. 6. It is observed that the CCEO algorithm achieves exactly the same performance as the known optimal solution in [20], and the proposed TS scheme provides closeto-optimal and significantly better performance than the MPC solution, especially when $\gamma$ is large and the caching capacity $M$ is small. The MPC solution is the worst caching scheme because it ignores the content diversity which is particularly important when the content popularity is more uniform. Fig. 7 shows the SCDP with different content sizes $\eta$. It is found that the SCDP of the TS scheme is closer to the optimum when the $\eta / T$ is large. However, as the bit rate of each content $\eta / T$ increases, both TS and MPC schemes become very close to the optimal solution. 


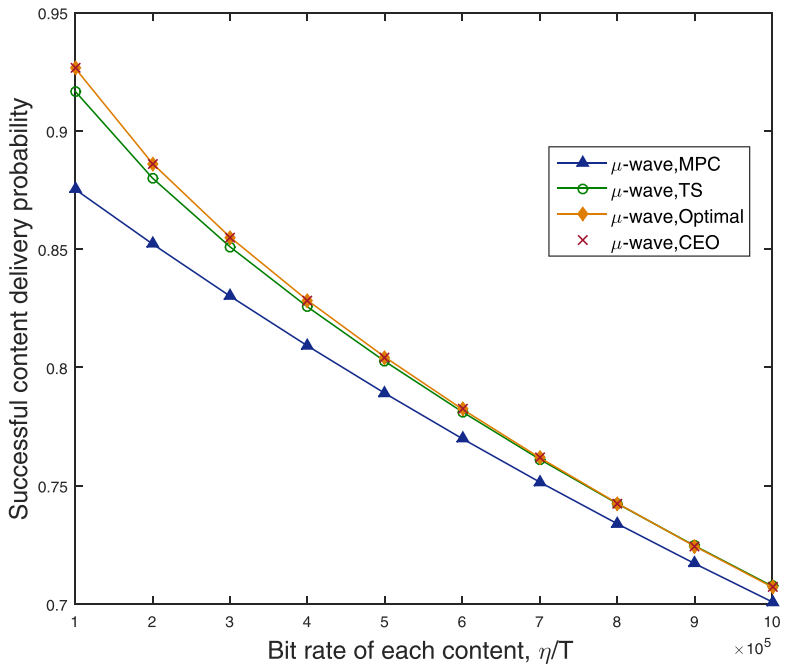

Fig. 7. Successful content delivery probability for $\mu$-wave single antennas, $\gamma=0.6$.

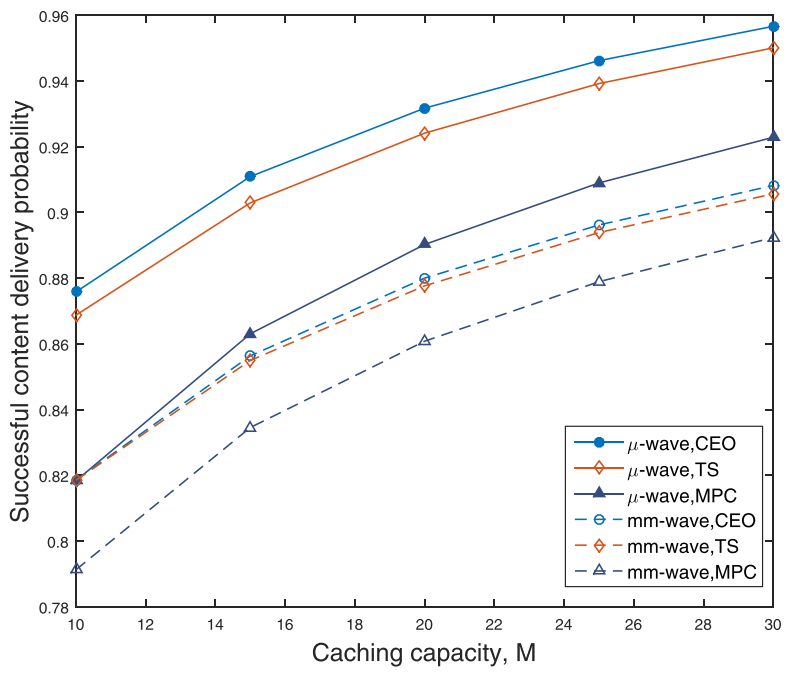

Fig. 8. The impact of $M$ on the successful content delivery probability, $\gamma=1.5$.

Fig. 8 shows the SCDP comparison of various systems with different caching capacities $M$. It shows that both of the proposed content placement schemes perform consistently better than MPC, especially for the $60 \mathrm{GHz} \mathrm{mm}$-wave, the SCDP of the TS scheme is close to that of the CCEO algorithm. The results also indicate that $\mu$-wave always has a superior performance than the $60 \mathrm{GHz} \mathrm{mm}$-wave with the same SBS density of $600 / \mathrm{km}^{2}$.

Fig. 9 shows the SCDP comparison of various systems versus the caching capacities $M$ with different content sizes. We generate a random set of of content size $S=\left\{s_{1}, \ldots, s_{j}, \ldots s_{J}\right\}$, where $s_{j}$ denotes the content size of $f_{j}$. For simplicity, $s_{j}$ is chosen to be 1 or 2 with equal probability of 0.5 in our simulation. The caching probability satisfies $\sum_{j=1}^{J} b_{j} \times s_{j} \leq M$. It is shown that in the unequal-size content case, CEO still greatly outperforms MPC, following a similar trend as the equal-size content case.
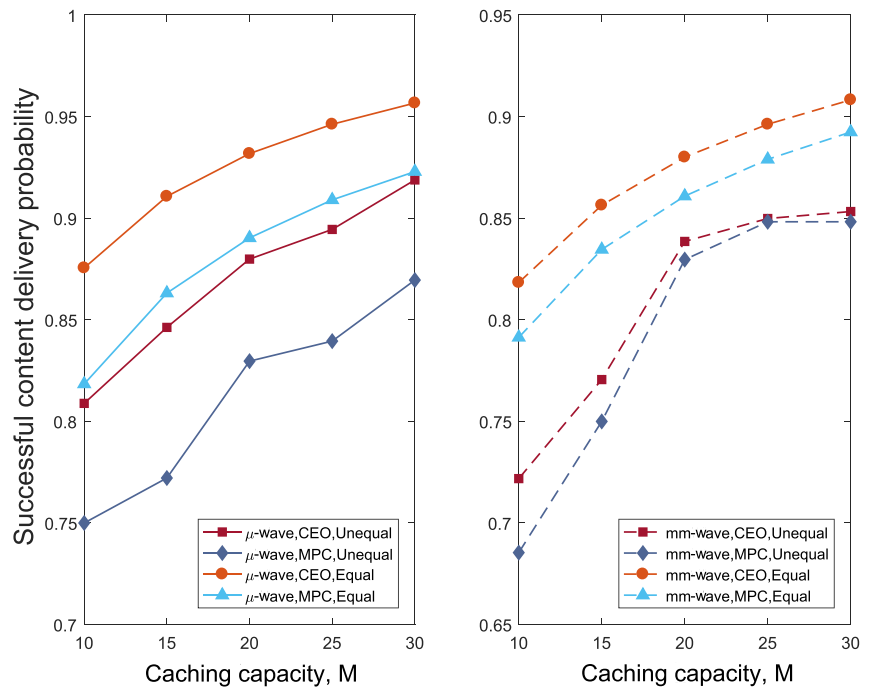

Fig. 9. The impact of $M$ on the successful content delivery probability with unequal content size, $\gamma=1.5$.

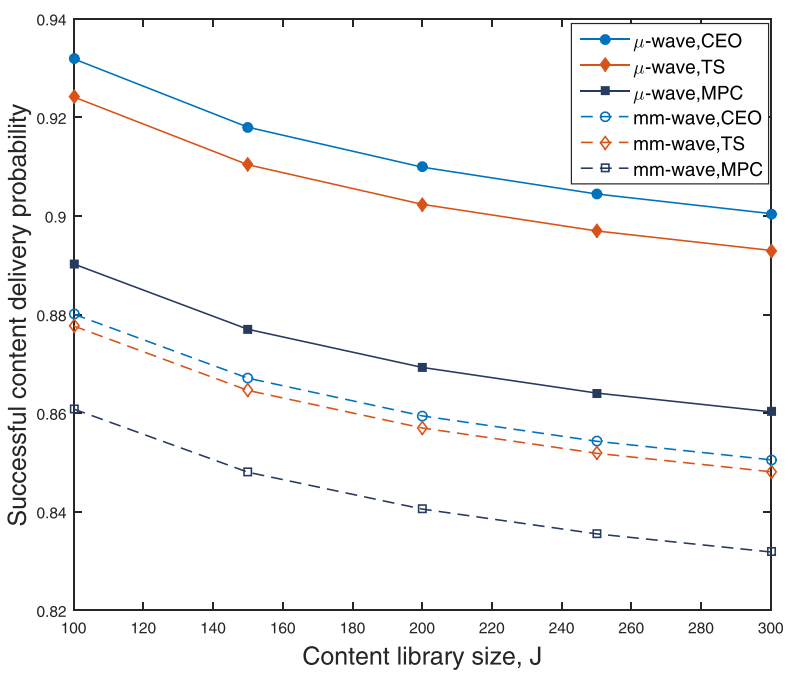

Fig. 10. The impact of $J$ on the successful content delivery probability, $\gamma=1.5, M=20$.

Fig. 10 studies the impact of content library size on SCDPs of different systems. It is seen that as the library size $J$ increases, the SCDP drops rapidly. The gap between the proposed content placement schemes and the MPC scheme remain stabilized when the library size increases.

Fig. 11 compares the SCDPs for the two proposed content placement schemes against Zipf exponent $\gamma$. It can be seen that the SCDP increases with $\gamma$ because caching is more effective when the content reuse is high. In the high- $\gamma$ regime of both $\mu$-wave and mm-wave systems, the content request probabilities for the first few most popular content are large, and SCDPs of both proposed placement schemes almost coincide. It is noteworthy that the proposed TS placement scheme achieves performance close to the CCEO algorithm, especially in the $\mu$-wave system and at low and high $\gamma$ regimes.

Finally, we investigate the cache-density tradeoff and its implication on the comparison of $\mu$-wave and mm-wave 


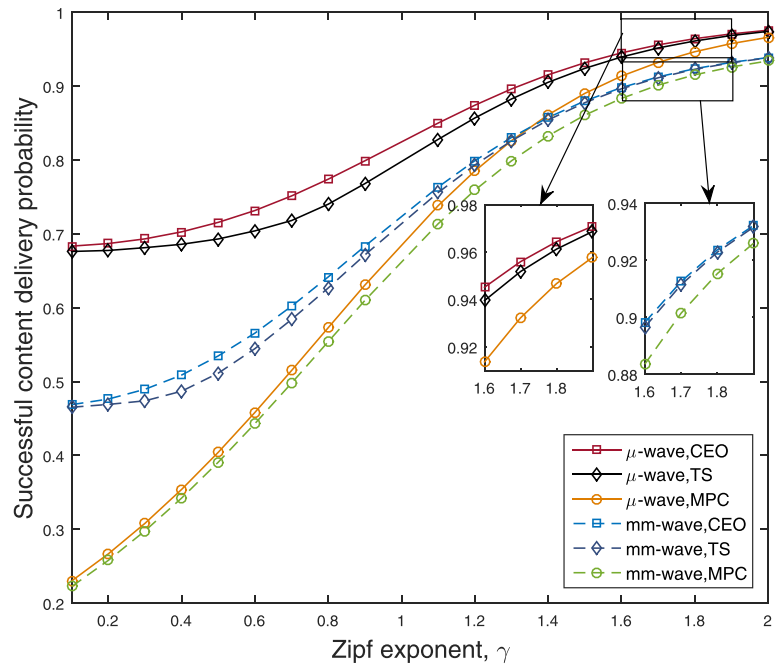

Fig. 11. The impact of Zipf exponent $\gamma$ on the successful content delivery probability.

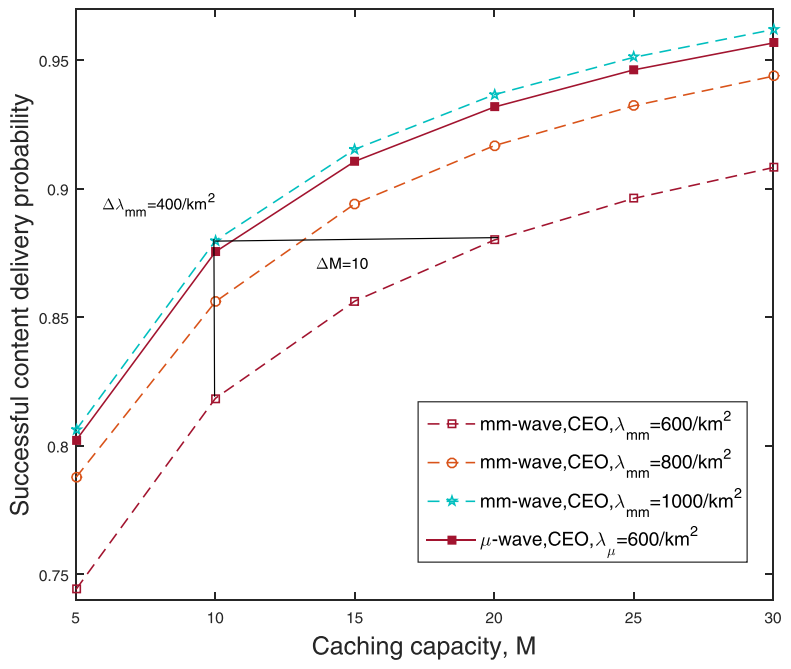

Fig. 12. Cache-density tradeoff, $\gamma=1.5$.

systems. The CEO placement scheme is used. Fig. 12 demonstrates the SCDPs with different caching capacity $M$, SBS densities $\lambda_{\mu}$ and $\lambda_{\mathrm{mm}}$. It is also observed that the $\mu$-wave channel is usually better than the mm-wave channel when $\lambda=600 / \mathrm{km}^{2}$, so with the same SBS density, $\mu$-wave achieves higher SCDP. To achieve performance comparable to that of the $\mu$-wave system with SBS density of $600 / \mathrm{km}^{2}$, the $\mathrm{mm}$-wave system needs to deploy SBSs with a much higher density of $1000 / \mathrm{km}^{2}$, but the extra density of $\triangle \lambda_{\mathrm{mm}}=400$ $/ \mathrm{km}^{2}$ is too costly to afford. Fortunately, by increasing the caching capacity from 10 to 20 , the mm-wave system can achieve the same SCDP of $91 \%$ as the $\mu$-wave system while keeping the same density of $600 / \mathrm{km}^{2}$. This result shows great promise of cache-enabled small cell systems because it is possible to trade off the relatively cheap storage for reduced expensive infrastructure.

\section{CONCLUSION}

In this paper, we have investigated the performance of caching in $\mu$-wave and mm-wave multi-antenna dense networks to improve the efficiency of content delivery. Using stochastic geometry, we have analyzed the successful content delivery probabilities and demonstrated the impact of various system parameters. We designed two novel caching schemes to maximize the successful content delivery probability with moderate to low complexities. The proposed CCEO algorithm can achieve near-optimal performance while the proposed TS scheme demonstrates performance close to CCEO with further reduced complexity. An important implication of this work is that to reduce the performance gap between the $\mu$-wave and $\mathrm{mm}$-wave systems, increasing caching capacity is a low-cost and effective solution compared to the traditional measures such as using more antennas or increasing SBS density. As a promising future direction, to study cooperative caching in a multi-band $\mu$-wave and mm-wave system could further reap the benefits of both systems.

\section{APPENDIX A}

Proof of THEOREM 1

Based on (5), $\mathcal{P}_{\mathrm{SCD}}^{\mu}$ is calculated as

$$
\begin{aligned}
\mathcal{P}_{\mathrm{SCD}}^{\mu} & =\sum_{j=1}^{J} a_{j} \operatorname{Pr}\left(\frac{P_{\mu} h_{j}^{\mu} L\left(\left|X_{j}^{\mu}\right|\right)}{\mathcal{I}_{j}^{\mu}+\overline{\mathcal{I}}_{j}^{\mu}+\sigma_{\mu}^{2}}>\varphi_{\mu}\right) \\
& =\sum_{j=1}^{J} a_{j} \int_{0}^{\infty} \underbrace{\operatorname{Pr}\left(\frac{P_{\mu} h_{j}^{\mu} L(x)}{\mathcal{I}_{j}^{\mu}+\overline{\mathcal{I}}_{j}^{\mu}+\sigma_{\mu}^{2}}>\varphi_{\mu}\right)}_{P_{\mathrm{cov}}^{\mu}\left(x, b_{j}\right)} f_{\left|X_{j}^{\mu}\right|}(x) d x,
\end{aligned}
$$

where $P_{\mathrm{cov}}^{\mu}\left(x, b_{j}\right)$ is the conditional coverage probability, and $f_{\left|X_{j}^{\mu}\right|}(x)$ is the PDF of the distance $\left|X_{j}^{\mu}\right|$. Then, we derive $P_{\text {cov }}^{\mu}\left(x, b_{j}\right)$ as

$$
\begin{aligned}
& P_{\mathrm{cov}}^{\mu}\left(x, b_{j}\right) \\
& =\operatorname{Pr}\left(\frac{P_{\mu} h_{j}^{\mu} \beta_{\mu} x^{-\alpha_{\mu}}}{\mathcal{I}_{j}^{\mu}+\overline{\mathcal{I}}_{j}^{\mu}+\sigma_{\mu}^{2}}>\varphi_{\mu}\right) \\
& =\int_{0}^{\infty} \operatorname{Pr}\left(h_{j}^{\mu}>\frac{\varphi_{\mu}\left(\tau+\sigma_{\mu}^{2}\right) x^{\alpha_{\mu}}}{P_{\mu} \beta_{\mu}}\right) d \operatorname{Pr}\left(\mathcal{I}_{\text {total }} \leq \tau\right) \\
& =\int_{0}^{\infty} e^{-\frac{\left(\tau+\sigma_{\mu}^{2}\right) \varphi_{\mu} x^{\alpha_{\mu}}}{P_{\mu} \beta_{\mu}}} \sum_{n=0}^{N_{\mu}-1} \frac{\left(\frac{\left(\tau+\sigma_{\mu}^{2}\right) \varphi_{\mu} x^{\alpha \mu}}{P_{\mu} \beta_{\mu}}\right)^{n}}{n !} d \operatorname{Pr}\left(\mathcal{I}_{\text {total }} \leq \tau\right)
\end{aligned}
$$

where $\mathcal{I}_{\text {total }}=\mathcal{I}_{j}^{\mu}+\overline{\mathcal{I}}_{j}^{\mu}$. Note that

$$
\begin{aligned}
& \left.\frac{d^{n}\left(\exp \left(-\frac{\left(\tau+\sigma_{\mu}^{2}\right) \varphi_{\mu} \nu}{P_{\mu} \beta_{\mu}}\right)\right)}{d \nu^{n}}\right|_{\nu=x^{\alpha \mu}} \\
& \quad=\left(-\frac{\left(\tau+\sigma_{\mu}^{2}\right) \varphi_{\mu}}{P_{\mu} \beta_{\mu}}\right)^{n} \exp \left(-\frac{\left(\tau+\sigma_{\mu}^{2}\right) \varphi_{\mu} \nu}{P_{\mu} \beta_{\mu}}\right) .
\end{aligned}
$$


By using (A.3), (A.2) can be rewritten as

$$
\begin{aligned}
& P_{\mathrm{cov}}^{\mu}\left(x, b_{j}\right) \\
& =\sum_{n=0}^{N_{\mu}-1} \frac{x^{n \alpha_{\mu}}}{n !(-1)^{n}} \\
& \times\left.\frac{d^{n}\left[\exp \left(-\frac{\nu \varphi_{\mu} \sigma_{\mu}^{2}}{P_{\mu} \beta_{\mu}}\right) \mathcal{L}_{\mathcal{I}_{j}^{\mu}}\left(\frac{\varphi_{\mu} \nu}{P_{\mu} \beta_{\mu}}\right) \mathcal{L}_{\overline{\mathcal{I}}_{j}^{\mu}}\left(\frac{\varphi_{\mu} \nu}{P_{\mu} \beta_{\mu}}\right)\right]}{d \nu^{n}}\right|_{\nu=x^{\alpha_{\mu}}},
\end{aligned}
$$

where $\mathcal{L}_{\mathcal{I}_{j}^{\mu}}(\cdot)$ is the Laplace transform of the PDF $\mathcal{I}_{j}^{\mu}$, and $\mathcal{L}_{\overline{\mathcal{I}}_{j}^{\mu}}(\cdot)$ is the Laplace transform of the $\operatorname{PDF} \overline{\mathcal{I}}_{j}^{\mu}$. Then $\mathcal{L}_{\mathcal{I}_{j}^{\mu}}(s)$ is given by

$$
\begin{aligned}
& \mathcal{L}_{\mathcal{I}_{j}^{\mu}}(s) \\
& =\mathbb{E}_{\Phi_{j}^{\mu}}\left[\exp \left(-s \sum_{i \in \Phi_{j}^{\mu} \backslash o} P_{\mu} h_{i, o} L\left(\left|X_{i, o}\right|\right)\right)\right] \\
& =\mathbb{E}_{\Phi_{j}^{\mu}}\left[\prod_{i \in \Phi_{j}^{\mu} \backslash\{o\}} \mathbb{E}_{h_{i, o}}\left\{\exp \left(-s P_{\mu} h_{i, o} L\left(\left|X_{i, o}\right|\right)\right)\right\}\right] \\
& =\exp \left[-\int_{x}^{\infty}\left(1-\mathbb{E}_{h_{i, o}}\left\{\exp \left(-s P_{\mu} h_{i, o} \beta_{\mu} r^{-\alpha_{\mu}}\right)\right\}\right) 2 \pi b_{j} \lambda_{\mu} r d r\right] \\
& =\exp \left[-2 \pi b_{j} \lambda_{\mu} \int_{x}^{\infty}\left(1-\frac{1}{1+s P_{\mu} \beta r^{-\alpha_{\mu}}}\right) r d r\right] .
\end{aligned}
$$

Likewise, $\mathcal{L}_{\overline{\mathcal{I}}_{j}^{\mu}}(s)$ is given by

$$
\begin{aligned}
\mathcal{L}_{\overline{\mathcal{I}}_{j}^{\mu}}(s)=\mathbb{E}_{\overline{\mathcal{I}}_{j}^{\mu}}\left[\exp \left(-s \sum_{k \in \bar{\Phi}_{j}^{\mu}} P_{\mu} h_{k, o} L\left(\left|X_{k, o}\right|\right)\right)\right] \\
=\mathbb{E}_{\overline{\mathcal{I}}_{j}^{\mu}}\left[\prod_{k \in \overline{\mathcal{I}}_{j}^{\mu}} \mathbb{E}_{h_{k, o}}\left\{\exp \left(-s P_{\mu} h_{k, o} L\left(\left|X_{k, o}\right|\right)\right)\right\}\right] \\
=\exp \left[-\int_{0}^{\infty}\left(1-\mathbb{E}_{h_{k, o}}\left\{\exp \left(-s P_{\mu} h_{k, o} \beta_{\mu} r^{-\alpha_{\mu}}\right)\right\}\right)\right. \\
\\
\times \exp \left[-2 \pi\left(1-b_{j}\right) \lambda_{\mu} r d r\right] \\
\\
\left.\times\left(1-\frac{1}{1+s P_{\mu} \beta_{\mu} r^{-\alpha_{\mu}}}\right) r d r\right] .
\end{aligned}
$$

Substituting (A.5) and (A.6) into (A.4), after some manipulations, we can obtain the desired result (10).

\section{APPENDIX B}

PROOF OF THEOREM 2

Based on (4) and (6), the SCDP for a LOS mm-wave link can be derived as

$$
\begin{aligned}
\mathcal{P}_{\mathrm{SCD}}^{\mathrm{mm}, \mathrm{L}}= & \int_{0}^{D_{L}} \operatorname{Pr}\left(\frac{P_{\mathrm{mm}} G_{\mathrm{mm}} \beta_{\mathrm{mm}} y^{-\alpha_{\mathrm{L}}}}{\sigma_{\mathrm{mm}}^{2}}>\varphi_{\mathrm{mm}}\right) f_{\left|Y_{j}^{\mathrm{mm}}\right|}(y) d y \\
= & \mathbb{1}\left(D_{L}<d_{\mathrm{L}}\right) \int_{0}^{D_{L}} f_{\mid Y_{j}^{\mathrm{mm} \mid}}(y) d y \\
& +\mathbb{1}\left(D_{L}>d_{\mathrm{L}}\right) \int_{0}^{d_{\mathrm{L}}} f_{\left|Y_{j}^{\mathrm{mm}}\right|}(y) d y \\
= & 1-e^{-\left(\min \left(D_{L}, d_{\mathrm{L}}\right)\right)^{2} \pi b_{j} \lambda_{\mathrm{mm}}},
\end{aligned}
$$

where $d_{\mathrm{L}}=\left(\frac{P_{\mathrm{mm}} G_{\mathrm{mm}} \beta_{\mathrm{mm}}}{\varphi_{\mathrm{mm}} \sigma_{\mathrm{mm}}^{2}}\right)^{\frac{1}{\alpha_{\mathrm{L}}}}, f_{\left|Y_{j}^{\mathrm{mm}}\right|}(y)$ is the PDF of the distance $\left|Y_{j}^{\mathrm{mm}}\right|$ between a typical user and its serving mm-wave SBS, which is given by [36]

$$
f_{\mid Y_{j}^{\mathrm{mm} \mid}}(y)=2 \pi b_{j} \lambda_{\mathrm{mm}} y e^{-\pi b_{j} \lambda_{\mathrm{mm}} y^{2}}, \quad y \geq 0 .
$$

Similarly, the SCDP for a NLOS mm-wave link can be derived as

$$
\begin{aligned}
\mathcal{P}_{\mathrm{SCD}}^{\mathrm{mm}, \mathrm{N}} & =\int_{D_{L}}^{\infty} \operatorname{Pr}\left(\frac{P_{\mathrm{mm}} G_{\mathrm{mm}} \beta_{\mathrm{mm}} y^{-\alpha_{\mathrm{N}}}}{\sigma_{\mathrm{mm}}^{2}}>\varphi_{\mathrm{mm}}\right) f_{\left|Y_{j}^{\mathrm{mm} \mid}\right|}(y) d y \\
& =\mathbb{1}\left(D_{L}<d_{\mathrm{N}}\right) \int_{D_{L}}^{d_{\mathrm{N}}} f_{\left|Y_{j}^{\mathrm{mm}}\right|}(y) d y \\
& =e^{-D_{L}^{2} \pi b_{j} \lambda_{\mathrm{mm}}}-e^{-\left(\max \left(D_{L}, d_{\mathrm{N}}\right)\right)^{2} \pi b_{j} \lambda_{\mathrm{mm}}},
\end{aligned}
$$

where $d_{\mathrm{N}}=\left(\frac{P_{\mathrm{mm}} G_{\mathrm{mm}} \beta_{m m}}{\varphi_{\mathrm{mm}} \sigma_{\mathrm{mm}}^{2}}\right)^{\frac{1}{\alpha_{\mathrm{N}}}}$. Thus, we obtain the SCDP expressions for a LOS/NLOS mm-wave link.

\section{APPENDIX C}

PROOF OF THEOREM 3

Let $f_{1}(\varepsilon)$ denote the objective function of the problem (30). We can obtain the first-order and the second-order derivatives of $f_{1}(\varepsilon)$ with respect to (w.r.t.) $\varepsilon$ as

$$
\begin{aligned}
\frac{\partial f_{1}(\varepsilon)}{\partial \varepsilon}= & \frac{1}{J^{1-\gamma}-1}\left[\left(\ell_{o}^{\mu}-1\right)(1-\gamma) \varepsilon^{-\gamma}\right. \\
& \left.+(1-\gamma)\left(1-\frac{1}{\varpi}\right)\left(\varepsilon+\frac{(1-\varepsilon)}{\varpi}\right)^{-\gamma}\right]
\end{aligned}
$$

and

$$
\begin{aligned}
& \frac{\partial^{2} f_{1}(\varepsilon)}{\partial \varepsilon^{2}} \\
& =\frac{1}{J^{1-\gamma}-1}(1-\gamma)(-\gamma) \\
& \quad \times\left[\left(\ell_{o}^{\mu}-1\right) \varepsilon^{-\gamma-1}+\left(1-\frac{1}{\varpi}\right)^{2}\left(\varepsilon+\frac{(1-\varepsilon)}{\varpi}\right)^{-\gamma-1}\right],
\end{aligned}
$$

respectively. Note that $\ell_{o}^{\mu} \geq 1$ and $\frac{1-\gamma}{J^{1-\gamma}-1}>0$, so we get $\frac{\partial^{2} f_{1}(\varepsilon)}{\partial \varepsilon^{2}} \leq 0$, which means that $f_{1}(\varepsilon)$ is a concave function w.r.t. $\varepsilon$. By setting $\frac{\partial f_{1}(\varepsilon)}{\partial \varepsilon}$ to zero, we obtain the stationary point as

$$
\varepsilon_{o}=\left(\left(\left(\frac{\ell_{o}^{\mu}-1}{\varpi^{-1}-1}\right)^{-1 / \gamma}-1\right) \varpi+1\right)^{-1}
$$

Note that $0 \leq \varpi \leq 1$, and $\frac{\ell_{o}^{\mu}-1}{\varpi^{-1}-1} \geq 0$, we have $\varepsilon_{o} \geq 0$. To obtain the optimal $\varepsilon^{*}$, we need to consider the following cases:

- Case 1: $0 \leq \varepsilon_{o}<1$. In this case, the optimal solution of the problem (30) is $\varepsilon^{*}=\varepsilon_{0}$.

- Case 2: $\varepsilon_{o} \geq 1$. In this case, $\frac{\partial f_{1}(\varepsilon)}{\partial \varepsilon} \geq 0$ for $\varepsilon \in[0,1]$, and thus the optimal solution of the problem (30) is $\varepsilon^{*}=1$.

Based on the above cases, we obtain (31) and complete the proof. 


\section{APPENDIX D}

Newton's Method to Optimize $\varpi$ In (32)

We propose Newton's Method to solve the non-convex problem (32) with fast convergence. Based on (28), the firstorder derivative of $\widetilde{\mathcal{P}}_{\mathrm{SCD}}^{\mu}$ is given by

$$
\begin{aligned}
& \frac{\partial \widetilde{\mathcal{P}}_{\mathrm{SCD}}^{\mu}}{\partial \varpi} \\
& =\mathcal{P}_{j, \mathrm{SCD}}^{\mu}(1) \frac{M^{1-\gamma}}{J^{1-\gamma}-1}(1-\gamma) \varepsilon_{o}^{-\gamma} \frac{\partial \varepsilon_{o}}{\partial \varpi} \\
& \quad+\frac{\partial \mathcal{P}_{j, \mathrm{SCD}}^{\mu}(\varpi)}{\partial \varpi} \frac{M^{1-\gamma}}{J^{1-\gamma}-1}\left[\left(\varepsilon_{o}+\frac{\left(1-\varepsilon_{o}\right)}{\varpi}\right)^{1-\gamma}-\varepsilon_{o}^{1-\gamma}\right] \\
& \quad+\mathcal{P}_{j, \mathrm{SCD}}^{\mu}(\varpi) \frac{M^{1-\gamma}}{J^{1-\gamma}-1}(1-\gamma)\left\{-\varepsilon_{o}^{-\gamma} \frac{\partial \varepsilon_{o}}{\partial \varpi}\right. \\
& \left.\quad+\left(\varepsilon_{o}+\frac{\left(1-\varepsilon_{o}\right)}{\varpi}\right)^{-\gamma}\left[\frac{\partial \varepsilon_{o}}{\partial \varpi}\left(1-\varpi^{-1}\right)+\frac{\varepsilon_{o}-1}{\varpi^{2}}\right]\right\},
\end{aligned}
$$

where

$$
\begin{aligned}
\frac{\partial \varepsilon_{o}}{\partial \varpi}=-\left(\varepsilon_{o}\right)^{2} & \left(\left(\frac{\ell_{o}-1}{\varpi^{-1}-1}\right)^{-1 / \gamma}-1\right. \\
& \left.-(\gamma \varpi)^{-1}\left(\ell_{o}-1\right)^{-\frac{1}{\gamma}}\left(\varpi^{-1}-1\right)^{\frac{1}{\gamma}-1}\right),
\end{aligned}
$$

and

$$
\begin{aligned}
\frac{\partial \mathcal{P}_{j, \mathrm{SCD}}^{\mu}(\varpi)}{\partial \varpi}=\int_{0}^{\infty} & P_{\operatorname{cov}}(x)\left(2 \pi \lambda_{\mu} x e^{-\pi \varpi \lambda_{\mu} x^{2}}\right. \\
& \left.-2 \pi^{2} \varpi \lambda_{\mu}^{2} x^{3} e^{-\pi \varpi \lambda_{\mu} x^{2}}\right) d x,
\end{aligned}
$$

respectively. In (D.3), to simplify the computation, we let $P_{\text {cov }}(x) \approx P_{\text {cov }}^{\mu}(x, 0)$, based on the fact that the interference $\mathcal{I}_{j}^{\mu}+\overline{\mathcal{I}}_{j}^{\mu}$ can be approximated as $\sum_{k \in \Phi_{\mu}} P_{\mu} h_{k, o} L\left(\left|X_{k, o}\right|\right)$, particularly in the dense small cell scenarios [44]. Similarly, the second-order derivative of $\widetilde{\mathcal{P}}_{\mathrm{SCD}}^{\mu}(\eta, T)$ is given by

$$
\begin{aligned}
\frac{\partial^{2} \widetilde{\mathcal{P}}_{\mathrm{SCD}}^{\mu}}{\partial \varpi^{2}} & =\mathcal{P}_{j, \mathrm{SCD}}^{\mu}(1) \frac{M^{1-\gamma}}{J^{1-\gamma}-1}(1-\gamma) \varepsilon_{o}^{-\gamma}\left(-\gamma \varepsilon_{o}^{-1}\left(\frac{\partial \varepsilon_{o}}{\partial \varpi}\right)^{2}+\frac{\partial^{2} \varepsilon_{o}}{\partial \varpi^{2}}\right) \\
& +\frac{\partial^{2} \mathcal{P}_{j, \mathrm{SCD}}^{\mu}(\varpi)}{\partial \varpi^{2}} \frac{M^{1-\gamma}}{J^{1-\gamma}-1}\left[\left(\varepsilon_{o}+\frac{\left(1-\varepsilon_{o}\right)}{\varpi}\right)^{1-\gamma}-\varepsilon^{1-\gamma}\right] \\
& +2 \frac{\partial \mathcal{P}_{j, \mathrm{SCD}}^{\mu}(\varpi)}{\partial \varpi} \frac{M^{1-\gamma}}{J^{1-\gamma}-1}(1-\gamma)\left\{-\varepsilon_{o}^{-\gamma} \frac{\partial \varepsilon_{o}}{\partial \varpi}\right. \\
& \left.+\left(\varepsilon_{o}+\frac{\left(1-\varepsilon_{o}\right)}{\varpi}\right)^{-\gamma}\left[\frac{\partial \varepsilon_{o}}{\partial \varpi}\left(1-\varpi^{-1}\right)+\frac{\varepsilon_{o}-1}{\varpi^{2}}\right]\right\} \\
& +\mathcal{P}_{j, \mathrm{SCD}}^{\mu}(\varpi) \frac{M^{1-\gamma}}{J^{1-\gamma}-1}(1-\gamma)\left\{\gamma \varepsilon_{o}^{-\gamma-1} \frac{\partial \varepsilon_{o}}{\partial \varpi}-\varepsilon_{o}^{-\gamma} \frac{\partial^{2} \varepsilon_{o}}{\partial \varpi^{2}}\right. \\
& -\gamma\left(\varepsilon_{o}+\frac{\left(1-\varepsilon_{o}\right)}{\varpi}\right)^{-\gamma-1}\left[\frac{\partial \varepsilon_{o}}{\partial \varpi}\left(1-\varpi^{-1}\right)+\frac{\varepsilon_{o}-1}{\varpi^{2}}\right]^{2} \\
& +\left(\varepsilon_{o}+\frac{\left(1-\varepsilon_{o}\right)}{\varpi}\right)^{-\gamma} \\
& \left.\times\left[\frac{\partial^{2} \varepsilon_{o}}{\partial \varpi^{2}}\left(1-\varpi \varpi^{-1}\right)+\frac{\partial \varepsilon_{o}}{\partial \varpi} \varpi^{-2}+\frac{\frac{\partial \varepsilon_{o}}{\partial \varpi} \varpi^{2}-2 \varpi\left(\varepsilon_{o}-1\right)}{\varpi^{4}}\right]\right\},
\end{aligned}
$$

where

$$
\begin{aligned}
\frac{\partial^{2} \varepsilon_{o}}{\partial \varpi^{2}}= & 2 \varepsilon_{o}^{-1}\left(\frac{\partial \varepsilon_{o}}{\partial \varpi}\right)^{2}-\varepsilon_{o}^{2}\left(\ell_{o}-1\right)^{-\frac{1}{\gamma}}\left[-\gamma^{-1} \varpi^{-2}\right. \\
& \times\left(\varpi^{-1}-1\right)^{\frac{1}{\gamma}-1}+\gamma^{-1} \varpi^{-2}\left(\varpi^{-1}-1\right)^{\frac{1}{\gamma}-1} \\
& \left.+\gamma^{-1}(\gamma \varpi)^{-3}\left(\frac{1}{\gamma}-1\right)\left(\varpi^{-1}-1\right)^{\frac{1}{\gamma}-2}\right]
\end{aligned}
$$

and

$$
\begin{aligned}
\frac{\partial^{2} \mathcal{P}_{j, \mathrm{SCD}}^{\mu}(\varpi)}{\partial \varpi^{2}}=\int_{0}^{\infty} P_{\mathrm{cov}}(x)( & -4 \pi^{2} \lambda_{\mu}^{2} x^{3} e^{-\pi \varpi \lambda_{\mu} x^{2}} \\
& \left.+2 \pi^{3} \varpi \lambda_{\mu}^{3} x^{5} e^{-\pi \varpi \lambda_{\mu} x^{2}}\right) d x .
\end{aligned}
$$

According to [42], the search direction in Newton method can be defined as

$$
\Delta \varpi=\frac{\partial \widetilde{\mathcal{P}}_{\mathrm{SCD}}^{\mu}}{\partial \varpi} /\left|\frac{\partial^{2} \widetilde{\mathcal{P}}_{\mathrm{SCD}}^{\mu}}{\partial \varpi^{2}}\right| .
$$

Then, $\varpi$ is iteratively updated according to

$$
\varpi(\varrho+1)=\left[\varpi(\varrho)+\delta_{2}(\varrho) \Delta \varpi\right]_{0}^{\frac{1}{e^{\mu}}},
$$

where $\varrho$ denotes the iteration index, $\ell_{o}^{\mu}=\frac{\mathcal{P}_{j, \mathrm{SCD}}^{\mu}(1)}{\mathcal{P}_{j, \mathrm{SCD}}^{\mu}(\varpi)}$ is already defined in (30), $\delta_{2}(\varrho)$ is the step size that can be determined by backtracking line search [45]. Thus, the optimal $\varpi^{*}$ can be obtained when reaching convergence.

\section{APPENDIX E \\ DeRIVATION OF THE SEARCh DiRECTION IN THE Newton Method to Optimize $\varpi$ IN (32)}

We use Newton Method to solve the problem (32). Here we only derive the search direction that involves the first and second-order derivative, and the rest is similar to Appendix D.

We first derive the first-order derivative. Similar to (D.1), we change $\frac{\partial \mathcal{P}_{j, \mathrm{SCD}}^{\mu}(\varpi)}{\partial \varpi}$ to $\frac{\partial \mathcal{P}_{j, \mathrm{SCD}}^{\mathrm{mm}}(\varpi)}{\partial \varpi}$ and get the result below:

$$
\begin{aligned}
\frac{\partial \mathcal{P}_{j, \mathrm{ST}}^{\mathrm{mm}}(\varpi)}{\partial \varpi} \\
=-D_{\mathrm{L}}^{2} \pi \lambda_{\mathrm{mm}} e^{-D_{\mathrm{L}}^{2} \pi \varpi \lambda_{\mathrm{mm}}} \\
\quad+\left(\min \left(D_{\mathrm{L}}, d_{\mathrm{L}}\right)\right)^{2} \pi \lambda_{\mathrm{mm}} e^{-\left(\min \left(D_{\mathrm{L}}, d_{\mathrm{L}}\right)\right)^{2} \pi \varpi \lambda_{\mathrm{mm}}} \\
\quad+\left(\max \left(D_{\mathrm{L}}, d_{\mathrm{N}}\right)\right)^{2} \pi \lambda_{\mathrm{mm}} e^{-\left(\max \left(D_{\mathrm{L}}, d_{\mathrm{N}}\right)\right)^{2} \pi \varpi \lambda_{\mathrm{mm}}} .
\end{aligned}
$$

Next we focus on the second-order derivative. Changing $\frac{\partial^{2} \mathcal{P}_{j, \mathrm{SCD}}^{\mu}(\varpi)}{\partial \varpi^{2}}$ to $\frac{\partial^{2} \mathcal{P}_{j, \mathrm{SCD}}^{\mathrm{mm}}(\varpi)}{\partial \varpi^{2}}$ in (D.4) leads to the following result:

$$
\begin{aligned}
\frac{\partial^{2} \mathcal{P}_{j, \mathrm{SCD}}^{\mathrm{mm}}(\varpi)}{\partial \varpi^{2}} & \\
= & D_{\mathrm{L}}^{4} \pi^{2} \lambda_{\mathrm{mm}}^{2} e^{-D_{\mathrm{L}}^{2} \pi \varpi \lambda_{\mathrm{mm}}} \\
& -\left(\min \left(D_{\mathrm{L}}, d_{\mathrm{L}}\right)\right)^{4} \pi^{2} \lambda_{\mathrm{mm}}^{2} e^{-\left(\min \left(D_{\mathrm{L}}, d_{\mathrm{L}}\right)\right)^{2} \pi \varpi \lambda_{\mathrm{mm}}} \\
& -\left(\max \left(D_{\mathrm{L}}, d_{\mathrm{N}}\right)\right)^{4} \pi^{2} \lambda_{\mathrm{mm}}^{2} e^{-\left(\max \left(D_{\mathrm{L}}, d_{\mathrm{N}}\right)\right)^{2} \pi \varpi \lambda_{\mathrm{mm}}} .
\end{aligned}
$$


Therefore, the search direction in Newton Method can be expressed as

$$
\Delta \varpi^{\mathrm{mm}}=\frac{\partial \widetilde{\mathcal{P}}_{\mathrm{SCD}}^{\mathrm{mm}}}{\partial \varpi} /\left|\frac{\partial^{2} \widetilde{\mathcal{P}}_{\mathrm{SCD}}^{\mathrm{mm}}}{\partial \varpi^{2}}\right| .
$$

\section{REFERENCES}

[1] Y. Zhu, G. Zheng, L. Wang, K.-K. Wong, and L. Zhao, "Performance analysis and optimization of cache-enabled small cell networks," in Proc. IEEE Global Commun. Conf. (GLOBECOM), Singapore, Dec. 2017, pp. 1-6.

[2] "Cisco visual networking index: Global mobile data traffic forecast update, 2016-2021 white paper," Cisco, San Jose, CA, USA, White Paper, Feb. 2017.

[3] C. Fang, F. R. Yu, T. Huang, J. Liu, and Y. Liu, "A survey of energyefficient caching in information-centric networking," IEEE Commun. Mag., vol. 52, no. 11, pp. 122-129, Nov. 2014.

[4] E. G. Larsson, T. L. Marzetta, H. Q. Ngo, and H. Yang. (Feb. 2017). "Antenna count for massive MIMO: $1.9 \mathrm{GHz}$ versus $60 \mathrm{GHz} . "$ [Online]. Available: https://arxiv.org/abs/1702.06111

[5] T. Bai and R. W. Heath, Jr. (2016). Comparing Massive MIMO and mmWave MIMO. [Online]. Available: http://ctw2014.ieeectw.org/slides/session3/heath-ctw_v6.pdf

[6] L. Wang, K.-K. Wong, S. Jin, G. Zheng, and R. W. Heath, Jr., "A new look at physical layer security, caching, and wireless energy harvesting for heterogeneous ultra-dense networks," IEEE Commun. Mag., to be published.

[7] J. Qiao, Y. He, and X. S. Shen, "Proactive caching for mobile video streaming in millimeter wave 5G networks," IEEE Trans. Wireless Commun., vol. 15, no. 10, pp. 7187-7198, Oct. 2016.

[8] J. G. Rois, B. Lorenzo, F. González-Castaño, and J. C. Burguillo, "Heterogeneous millimeter-wave/micro-wave architecture for $5 \mathrm{G}$ wireless access and backhauling," in Proc. Eur. Conf. Netw. Commun. (EuCNC), Oulu, Finland, Sep. 2016, pp. 179-184.

[9] O. Semiari, W. Saad, M. Bennis, and B. Maham. (2017). "Caching meets millimeter wave communications for enhanced mobility management in 5G networks." [Online]. Available: https://arxiv.org/abs/1701.05125

[10] B. Xia, C. Yang, and T. Cao, "Modeling and analysis for cache-enabled networks with dynamic traffic," IEEE Commun. Lett., vol. 20, no. 12, pp. 2506-2509, Dec. 2016.

[11] A. Liu and V. K. N. Lau, "Exploiting base station caching in MIMO cellular networks: Opportunistic cooperation for video streaming," IEEE Trans. Signal Process., vol. 63, no. 1, pp. 57-69, Jan. 2015.

[12] A. Liu and V. K. N. Lau, "Cache-enabled opportunistic cooperative MIMO for video streaming in wireless systems," IEEE Trans. Signal Process., vol. 62, no. 2, pp. 390-402, Jan. 2014.

[13] S. Yang, K.-H. Ngo, and M. Kobayashi, "Content delivery with coded caching and massive MIMO in 5G," in Proc. 9th Int. Symp. Turbo Codes Iterative Inf. Process. (ISTC), Brest, France, Sep. 2016, pp. 370-374.

[14] Z. Chen, J. Lee, T. Q. S. Quek, and M. Kountouris, "Cooperative caching and transmission design in cluster-centric small cell networks," IEEE Trans. Wireless Commun., vol. 16, no. 5, pp. 3401-3415, May 2017.

[15] G. Zheng, H. A. Suraweera, and I. Krikidis, "Optimization of hybrid cache placement for collaborative relaying," IEEE Commun. Lett., vol. 21, no. 2, pp. 442-445, Feb. 2017

[16] W. C. Ao and K. Psounis, "Distributed caching and small cell cooperation for fast content delivery," in Proc. ACM Int. Symp. Mobile Ad Hoc Netw. Comput., 2015, pp. 127-136.

[17] B. Blaszczyszyn and A. Giovanidis, "Optimal geographic caching in cellular networks," in Proc. IEEE Int. Conf. Commun. (ICC), London, U.K., Jun. 2015, pp. 3358-3363.

[18] B. Serbetci and J. Goseling, "On optimal geographical caching in heterogeneous cellular networks," in Proc. IEEE Wireless Commun. Netw. Conf. (WCNC), San Francisco, CA, USA, Mar. 2017, pp. 1-6.

[19] K. Li, C. Yang, Z. Chen, and M. Tao. (2016). "Optimization and analysis of probabilistic caching in $n$-tier heterogeneous networks." [Online] Available: https://arxiv.org/abs/1612.04030

[20] J. Wen, K. Huang, S. Yang, and V. O. K. Li, "Cache-enabled heterogeneous cellular networks: Optimal tier-level content placement," IEEE Trans. Wireless Commun., vol. 16, no. 9, pp. 5939-5952, Sep. 2017.

[21] D. Liu and C. Yang, "Caching policy toward maximal success probability and area spectral efficiency of cache-enabled HetNets," IEEE Trans. Commun., vol. 65, no. 6, pp. 2699-2714, Jun. 2017.
[22] W. Wen, Y. Cui, F.-C. Zheng, S. Jin, and Y. Jiang. (2017). "Random caching based cooperative transmission in heterogeneous wireless networks." [Online]. Available: https://arxiv.org/abs/1701.05761

[23] S. H. Chae and W. Choi, "Caching placement in stochastic wireless caching helper networks: Channel selection diversity via caching," IEEE Trans. Wireless Commun., vol. 15, no. 10, pp. 6626-6637, Jun. 2016.

[24] X. Yu, C. Li, J. Zhang, and K. B. Letaief, "A tractable framework for performance analysis of dense multi-antenna networks," in Proc. IEEE Int. Conf. Commun. (ICC), Paris, France, May 2017, pp. 1-6.

[25] R. Y. Rubinstein, "Optimization of computer simulation models with rare events," Eur. J. Oper. Res., vol. 99, no. 1, pp. 89-112, 1997.

[26] L. Breslau, P. Cao, L. Fan, G. Phillips, and S. Shenker, "Web caching and zipf-like distributions: Evidence and implications," in Proc. IEEE INFOCOM, New York, NY, USA, Mar. 1999, pp. 126-134.

[27] Service Requirements for the 5G System, document 3GPP TS 22.261, Mar. 2017.

[28] Y. Zhu, L. Wang, K.-K. Wong, S. Jin, and Z. Zheng, "Wireless power transfer in massive MIMO-aided HetNets with user association," IEEE Trans. Commun., vol. 64, no. 10, pp. 4181-4195, Oct. 2016.

[29] T. S. Rappaport et al., "Millimeter wave mobile communications for 5G cellular: It will work!" IEEE Access, vol. 1, pp. 335-349, 2013.

[30] O. El Ayach, S. Rajagopal, S. Abu-Surra, Z. Pi, and R. W. Heath, Jr., "Spatially sparse precoding in millimeter wave MIMO systems," IEEE Trans. Wireless Commun., vol. 13, no. 3, pp. 1499-1513, Mar. 2014.

[31] T. Bai and R. W. Heath, Jr., "Coverage and rate analysis for millimeterwave cellular networks," IEEE Trans. Wireless Commun., vol. 14, no. 2, pp. 1100-1114, Feb. 2015.

[32] J. Park, S.-L. Kim, and J. Zander, "Tractable resource management with uplink decoupled millimeter-wave overlay in ultra-dense cellular networks," IEEE Trans. Wireless Commun., vol. 15, no. 6, pp. 4362-4379, Jun. 2016.

[33] S. Singh, M. N. Kulkarni, A. Ghosh, and J. G. Andrews, "Tractable model for rate in self-backhauled millimeter wave cellular networks," IEEE J. Sel. Areas Commun., vol. 33, no. 10, pp. 2196-2211, Oct. 2015.

[34] K. Venugopal, M. C. Valenti, and R. W. Heath, Jr., "Device-to-device millimeter wave communications: Interference, coverage, rate, and finite topologies," IEEE Trans. Wireless Commun., vol. 15 , no. 9, pp. 6175-6188, Sep. 2016.

[35] J. G. Andrews, T. Bai, M. N. Kulkarni, A. Alkhateeb, A. K. Gupta, and R. W. Heath, Jr., "Modeling and analyzing millimeter wave cellular systems," IEEE Trans. Commun., vol. 65, no. 1, pp. 403-430, Jan. 2017.

[36] H.-S. Jo, Y. J. Sang, P. Xia, and J. G. Andrews, "Heterogeneous cellular networks with flexible cell association: A comprehensive downlink SINR analysis," IEEE Trans. Wireless Commun., vol. 11, no. 10, pp. 3484-3495, Oct. 2012.

[37] Z. I. Botev, D. P. Kroese, R. Y. Rubinstein, and P. L'Ecuyer, "The crossentropy method for optimization," in Machine Learning: Theory and Applications, vol. 31, V. Govindaraju and C. R. Rao, Eds. Chennai, India: Elsevier, 2013, pp. 35-59.

[38] Y. Zhang, C. Ji, W. Q. Malik, D. C. O'Brien, and D. J. Edwards, "Receive antenna selection for MIMO systems over correlated fading channel," IEEE Trans. Wireless Commun., vol. 8, no. 9, pp. 4393-4399, Sep. 2009

[39] M. Caserta and M. C. Nodar, "A cross entropy based algorithm for reliability problems," J. Heuristics, vol. 15, no. 5, pp. 479-501, 2009.

[40] R. Rubinstein, "The cross-entropy method for combinatorial and continuous optimization," Methodol. Comput. Appl. Probab., vol. 1, no. 2, pp. 127-190, Sep. 1999.

[41] M. Taghizadeh, K. Micinski, S. Biswas, C. Ofria, and E. Torng, "Distributed cooperative caching in social wireless networks," IEEE Trans. Mobile Comput., vol. 12, no. 6, pp. 1037-1053, Jun. 2013.

[42] W. Yu, T. Kwon, and C. Shin, "Multicell coordination via joint scheduling, beamforming, and power spectrum adaptation," IEEE Trans. Wireless Commun., vol. 12, no. 7, pp. 1-14, Jul. 2013.

[43] T. S. Rappaport, E. Ben-Dor, J. N. Murdock, and Y. Qiao, "38 GHz and $60 \mathrm{GHz}$ angle-dependent propagation for cellular \& peer-to-peer wireless communications," in Proc. IEEE Int. Conf. Commun. (ICC), Ottawa, ON, Canada, Jun. 2012, pp. 4568-4573.

[44] X. Lin, J. G. Andrews, and A. Ghosh, "Spectrum sharing for deviceto-device communication in cellular networks," IEEE Trans. Wireless Commun., vol. 13, no. 12, pp. 6727-6740, Dec. 2014.

[45] S. Boyd and L. Vandenberghe, Convex Optimization. Cambridge, U.K.: Cambridge Univ. Press, 2004. 


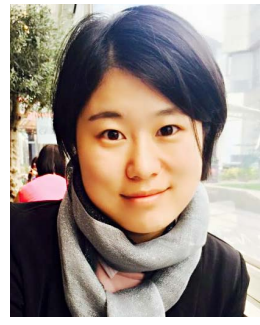

Yongxu Zhu received the M.S. degree from the Beijing University of Posts and Telecommunications and Dublin City University, in 2012 and 2013, respectively, and the $\mathrm{Ph} . \mathrm{D}$. degree in electrical engineering from University College London, in 2017. She is currently a Research Associate with Loughborough University. Her research interests are in the areas of energy harvesting wireless communications, wireless edge caching, mm-wave communications, heterogeneous cellular networks, massive MIMO, and physical-layer security.

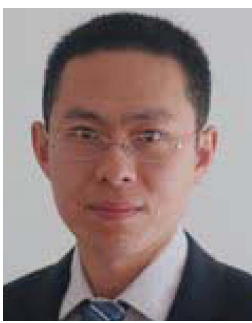

Gan Zheng (S'05-M'09-SM'12) received the B.Eng. and M.Eng. degrees in electronic and information engineering from Tianjin University, Tianjin, China, in 2002 and 2004, respectively, and the $\mathrm{Ph} . \mathrm{D}$. degree in electrical and electronic engineering from The University of Hong Kong in 2008. $\mathrm{He}$ is currently a Senior Lecturer with the Wolfson School of Mechanical, Electrical and Manufacturing Engineering, Loughborough University, U.K. His research interests include edge caching, full-duplex radio, wireless power transfer, cooperative communications, cognitive radio, and physical-layer security. He was a recipient of the 2013 IEEE Signal Processing Letters Best Paper Award, and also a recipient of the 2015 GLOBECOM Best Paper Award. He currently serves as an Associate Editor for the IEEE COMMUNICATIONS LETTERS.

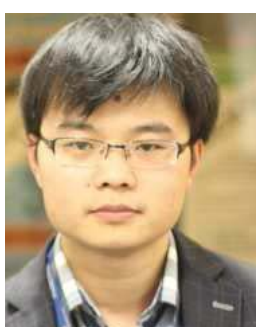

Lifeng Wang (M'15) is currently the Post-Doctoral Research Fellow with the Department of Electronic and Electrical Engineering, University College London. His research interests include massive MIMO, mm-wave, dense HetNets, edge caching, physical-layer security, and wireless energy harvesting. He was a recipient of the Exemplary Editor Certificates of the IEEE COMMUNICATIONS LETTERS in 2016 and 2017.

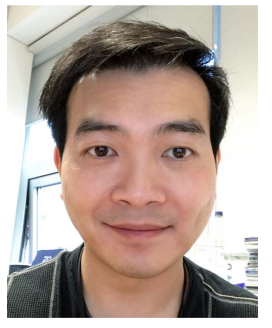

Kai-Kit Wong (M'01-SM'08-F'16) received the B.Eng., M.Phil., and Ph.D. degrees from The Hong Kong University of Science and Technology, Hong Kong, in 1996, 1998, and 2001, respectively, all in electrical and electronic engineering. After graduation, he took up academic and research positions at the University of Hong Kong, Lucent Technologies, Bell-Labs, Holmdel, NJ, USA, the Smart Antennas Research Group, Stanford University, Stanford, CA, USA, and the University of Hull, U.K. He is a Chair in Wireless Communications with the Department of Electronic and Electrical Engineering, University College London, U.K.

His current research centers around $5 \mathrm{G}$ and beyond mobile communications, including topics such as massive MIMO, full-duplex communications, mm-wave communications, edge caching and fog networking, physical layer security, wireless power transfer and mobile computing, V2X communications, and of course cognitive radios. There are also a few other unconventional research topics that he has set his heart on, including for example, fluid antenna communications systems and remote ECG detection. He was a co-recipient of the 2013 IEEE Signal Processing LetTers Best Paper Award, the 2000 IEEE VTS Japan Chapter Award at the IEEE Vehicular Technology Conference, Japan, in 2000, and a few other international best paper awards.

He is Fellow of IET and is also on the editorial board of several international journals. He has served as a Senior Editor for the IEEE COMMUNICATIONS LETTERS since 2012 and also for the IEEE WIRELESS COMMUNICATIONS LETTERS since 2016. He previously served as an Editor for the IEEE TRANSACTIONS ON WIRELESS COMMUNICATIONS from 2005 to 2011 and as an Associate Editor for the IEEE Signal Processing LeTters from 2009 to 2012. He was also a Guest Editor for the IEEE JSAC Special Issue on Virtual MIMO in 2013 and is currently a Guest Editor for the IEEE JSAC Special Issue on Physical Layer Security for 5G.

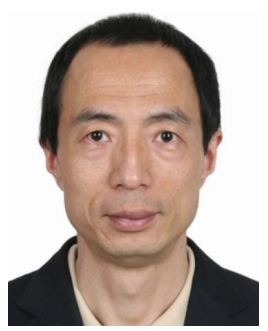

Liqiang Zhao (M'12) received the B.Sc. degree in electrical engineering from Shanghai Jiao Tong University, China, in 1992, and the M.Sc. degree in communications and information systems and the $\mathrm{Ph} . \mathrm{D}$. degree in information and communications engineering from Xidian University, in 2000 and 2003 , respectively. In 2005, he joined the School of Telecommunications Engineering, Xidian University, China, where he is currently developing his research and teaching activities as a Full Professor. His current research focuses on wireless communications, especially software defined network, network function virtualization, edge computing and caching, network slicing, energy harvesting, and nanonetwork. He has authored over 150 papers. He has hosted/participated in a great many research projects, such as the National Natural Science Foundation of China, 863 program, the EU FP6, FP7 plans, and the Huawei fund. 